\title{
Correlating ligand-to-metal charge transfer with voltage hysteresis in a Li-rich rock-salt compound exhibiting anionic redox
}

\author{
Biao Li ${ }^{1,2}$, Moulay Tahar Sougrati ${ }^{2,3}$, Gwenaëlle Rousse ${ }^{1,2,4}$, Anatolii V. Morozov ${ }^{5}$ Rémi Dedryvère ${ }^{2,6}$, \\ Antonella ladecola $\mathbb{1}^{2}$, Anatoliy Senyshyn ${ }^{7}$, Leiting Zhang $\mathbb{1}^{8}$, Artem M. Abakumov ${ }^{1}{ }^{5}$, \\ Marie-Liesse Doublet ${ }^{2,3}$ and Jean-Marie Tarascon ${ }^{1,2,4} \bowtie$
}

\begin{abstract}
Anionic redox is a double-edged sword for Li-ion cathodes because it offers a transformational increase in energy density that is also negated by several detrimental drawbacks to its practical implementation. Among them, voltage hysteresis is the most troublesome because its origin is still unclear and under debate. Herein, we tackle this issue by designing a prototypical Li-rich cation-disordered rock-salt compound $\mathrm{Li}_{1.17} \mathrm{Ti}_{0.33} \mathrm{Fe}_{0.5} \mathrm{O}_{2}$ that shows anionic redox activity and exceptionally large voltage hysteresis while exhibiting a partially reversible Fe migration between octahedral and tetrahedral sites. Through combined in situ and ex situ spectroscopic techniques, we demonstrate the existence of a non-equilibrium (adiabatic) redox pathway enlisting $\mathrm{Fe}^{3+} / \mathrm{Fe}^{4+}$ and $\mathrm{O}$ redox as opposed to the equilibrium (non-adiabatic) redox pathway involving sole $\mathrm{O}$ redox. We further show that the charge transfer from $\mathrm{O}(2 p)$ lone pair states to $\mathrm{Fe}(3 d)$ states involving sluggish structural distortion is responsible for voltage hysteresis. This study provides a general understanding of various voltage hysteresis signatures in the large family of Li-rich rock-salt compounds.
\end{abstract}

Re. echargeable Li-ion batteries offer great hopes for achieving higher energy density and meeting increasing future societal energy storage demands. The emergence of anionic redox opens a new direction towards the design of Li-rich positive electrodes (cathodes), $\mathrm{Li}_{1+x} \mathrm{TM}_{1-x} \mathrm{O}_{2}$ ( $\mathrm{TM}$ denotes transition metal, $0<x<1$ ), with high energy density, relying on cumulative cationic and anionic redox reactions ${ }^{1-4}$. The science underlying anionic redox has reached a great level of understanding owing to the wide family of Li-rich rock-salt structures that enlist either layered oxides $\left(\mathrm{Li}_{2} \mathrm{RuO}_{3}, \mathrm{Li}_{2} \mathrm{IrO}_{3} \text { and so on }\right)^{5-8}$ or isostructural $\mathrm{Li}_{2} \mathrm{TiS}_{3}$-based sulfides $^{9,10}$. Despite these fruitful understandings, a remaining practical issue regards how to utilize anionic redox in real-world applications. The well-known anionic-redox-based archetypical cathode, Li-rich nickel-manganese-cobalt oxide (Li-rich NMC), can deliver capacity as high as $300 \mathrm{~mA} \mathrm{~h} \mathrm{~g}^{-1}$. However, its commercialization is plagued by important issues, such as sluggish kinetics, voltage hysteresis and voltage fade ${ }^{11,12}$. Thus, further studies are urgently needed to get deeper insights into these issues so as to provide a cure that could help in bridging the gap between fundamental research and real applications.

It is well accepted that the voltage-fade problem is linked to irreversible cation migration, but there are still many debates regarding the origin of voltage hysteresis. Numerous researchers have proposed that voltage hysteresis is deeply rooted in reversible cation migration, which perturbs the Li site energies on lithium removal/ uptake $^{12-14}$. However, this view is challenged by a recent study claiming that highly reversible cation migration favours the mitigation of the redox asymmetry/voltage hysteresis in the compound
O2- $\mathrm{Li}_{x}\left(\mathrm{Li}_{02} \mathrm{Ni}_{02} \mathrm{Mn}_{0,6}\right) \mathrm{O}_{2}$ (ref. ${ }^{15}$ ). Other proposed mechanisms either rely on the energy penalty caused by the (re)formation/ breaking of $\mathrm{O}-\mathrm{O}$ dimers $^{16}$, redox inversion induced by a small charge-transfer bandgap ${ }^{17}$ or sluggish structural rearrangements through cycling as implied by micro-calorimetry study ${ }^{18}$. More recently, first-cycle hysteresis was elegantly linked to the formation of trapped molecular $\mathrm{O}_{2}$ favoured by irreversible cation migration in P2-type $\mathrm{Na}_{0.75}\left[\mathrm{Li}_{0.25} \mathrm{Mn}_{0.75}\right] \mathrm{O}_{2}$ and Li-rich NMC compounds ${ }^{19,20}$ although its persistence on cycling was not tackled. Obviously, further studies are thus desirable to clarify voltage hysteresis.

Interestingly, most of the aforementioned studies have overlooked the thermodynamic-kinetic aspects of anionic redox as a possible origin of voltage hysteresis. Ohmic resistance and Li-diffusion limitation are part of kinetic polarization that can be experimentally decoupled from other observables by slowing down the current rate, or investigated more elegantly using the galvanostatic intermittent titration technique (GITT). For classical insertion compounds, this polarization closes at zero current with the charge and discharge relaxed potentials nearly superimposing. By contrast, most anionic-redox electrodes show GITT profiles that hardly close the voltage gap ${ }^{11,21}$. This led researchers to propose 'quasi-static hysteresis' or even 'thermodynamic' originated hysteresis ${ }^{18,22}$. Another unexploited piece of information regarding voltage hysteresis is that its amplitude changes among various anionic-redox compounds, namely it increases from $\mathrm{Li}_{2} \mathrm{RuO}_{3}-$ to $\mathrm{Li}_{2} \mathrm{MnO}_{3}$ - and then to $\mathrm{Li}_{2} \mathrm{TiO}_{3}$-based phases.

In this Article, we investigate a Li-rich cation-disordered rock-salt compound, $0.4 \mathrm{Li}_{2} \mathrm{TiO}_{3}-0.6 \mathrm{LiFeO}_{2} \quad\left(\mathrm{Li}_{1.17} \mathrm{Ti}_{0.33} \mathrm{Fe}_{0.5} \mathrm{O}_{2}\right)$,

${ }^{1}$ Chimie du Solide-Energie, UMR 8260, Collège de France, Paris, France. ${ }^{2}$ Réseau sur le Stockage Electrochimique de l'Energie (RS2E), Amiens, France. ${ }^{3}$ ICGM, Univ Montpellier, CNRS, ENSCM, Montpellier, France. ${ }^{4}$ Sorbonne Université, Paris, France. ${ }^{5}$ Center for Energy Science and Technology, Skolkovo Institute of Science and Technology, Moscow, Russia. ${ }^{6}$ IPREM, E2S-UPPA, CNRS, University of Pau \& Pays Adour, Pau, France. ${ }^{7}$ Forschungsneutronenquelle Heinz Maier-Leibnitz (FRM II), Technische Universität München, Garching, Germany. ${ }^{8}$ Electrochemistry Laboratory, Paul Scherrer Institute, Villigen PSI, Switzerland. $凶_{e}$-mail: jean-marie.tarascon@college-de-france.fr 
denoted hereafter as LTFO, that combines anionic/cationic redox activity with a prominent voltage hysteresis of $\sim 1.4 \mathrm{~V}$. Although this compound shows a partially reversible Fe migration between octahedral and tetrahedral sites, we demonstrate that this specific feature is not the origin of voltage hysteresis. Instead, we prove that the sluggish reductive coupling mechanism involving a charge transfer from $\mathrm{O}(2 p)$ to $\mathrm{Fe}(3 d)$ states is responsible for the hysteresis. We also shed light on how the covalence/ionicity governs the charge-transfer kinetics in different compounds and discuss its role in voltage hysteresis.

\section{Results and discussion}

Structure and electrochemistry of LTFO. Electrochemically active well-crystallized LTFO powders containing particles with $30-50 \mathrm{~nm}$ diameters were obtained by a hydrothermal process, as detailed in the Methods section. The elemental nominal composition of the obtained powders was confirmed by inductively coupled plasma atomic emission spectroscopy (ICP-AES), while their homogeneity was verified by scanning transmission electron microscopy-energy dispersive X-ray spectroscopy (STEM-EDX) analysis (Supplementary Fig. 1) revealing the $\mathrm{Ti} / \mathrm{Fe}=0.40(2): 0.60(2)$ atomic ratio. Rietveld refinement from a synchrotron powder X-ray diffraction (SXRD) pattern (Fig. 1a and Supplementary Table 1a) shows that LTFO adopts a cation-disordered rock-salt structure $(F m \overline{3} m)$ while the [100] and [110] electron diffraction (ED) patterns (Fig. 2e and Supplementary Fig. 2a) indicate a structured diffuse scattering (labelled with arrows). This is evidence for cationic short-range ordering driven by the tendency for each $\mathrm{O}$ atom to be as homogeneously as possible surrounded by the TM and Li cations, like $\left[\mathrm{Li}_{3} \mathrm{TM}_{3}\right]$ clusters in ordered layered compounds.

The electrochemical behaviour of LTFO was tested versus Li between 2.0 and $4.8 \mathrm{~V}$ at a $\mathrm{C} / 10$ rate (Fig. 1b). On oxidation, the removal of Li occurs via a single-step plateau located at $\sim 4.25 \mathrm{~V}$ as opposed to two different (pseudo)plateaus on the subsequent discharge, with the higher one showing a huge voltage hysteresis of $\sim 1.4 \mathrm{~V}$. The large irreversibility of $\sim 0.3 \mathrm{Li}$ during the first cycle (Fig. 1b) arises not only from electrochemical decomposition of $\mathrm{Li}_{2} \mathrm{CO}_{3}$ surface species and oxygen release but also from kinetic limitations, as deduced by online electrochemical mass spectrometry (OEMS) and constant-current-constant-voltage (CCCV) experiments, respectively (Supplementary Fig. 3).

The huge voltage hysteresis of $\sim 1.4 \mathrm{~V}$ persists on further cycles, even when slowing down charge-discharge rates (Fig. 1c), indicating that this hysteretic behaviour is not simply nested in conventional kinetic polarizations caused by poor electronic/ionic conductivity. The hysteresis is shown to be path-independent by open-voltage-window experiments (Supplementary Fig. 4). This contrasts with the previously reported path-dependent voltage hysteresis in Li-rich NMC which led researchers to propose a 'thermodynamic' origin indirectly linked to cation migration ${ }^{13}$. Here we believe that our finding of path-independence in LTFO stems from the absence of interplay between cationic and anionic redox events, as will be shown later.

Partially reversible Fe migration. The LTFO long-range structural evolution on cycling was checked using an in situ X-ray diffraction (XRD) cell cycled at a C/10 rate and XRD patterns were collected for every change in lithium stoichiometry of $\sim 0.03$ (Supplementary Fig. 5). On charging, both (002) and (220) peaks initially shift to high angles and then shift back while their amplitudes progressively decrease. Such a two-stage feature in XRD is also consistent with the GITT curve at first charge unravelling two open-circuit-voltage (OCV) processes (Supplementary Fig. 6). The subsequent discharge and second charge show similar peak shifting and intensity evolution with high reversibility, as highlighted by the contour plots in Supplementary Fig. 5c. Overall, from the 2nd cycle and onwards

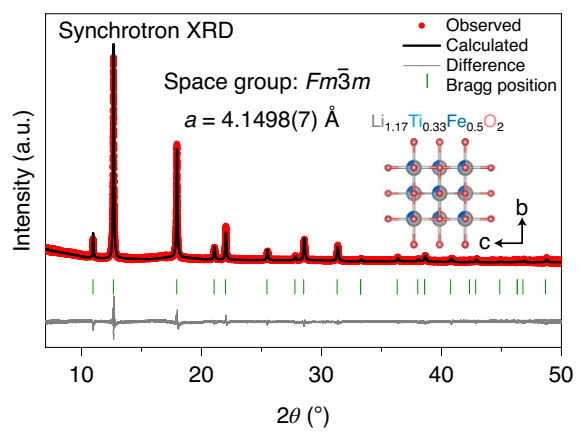

b
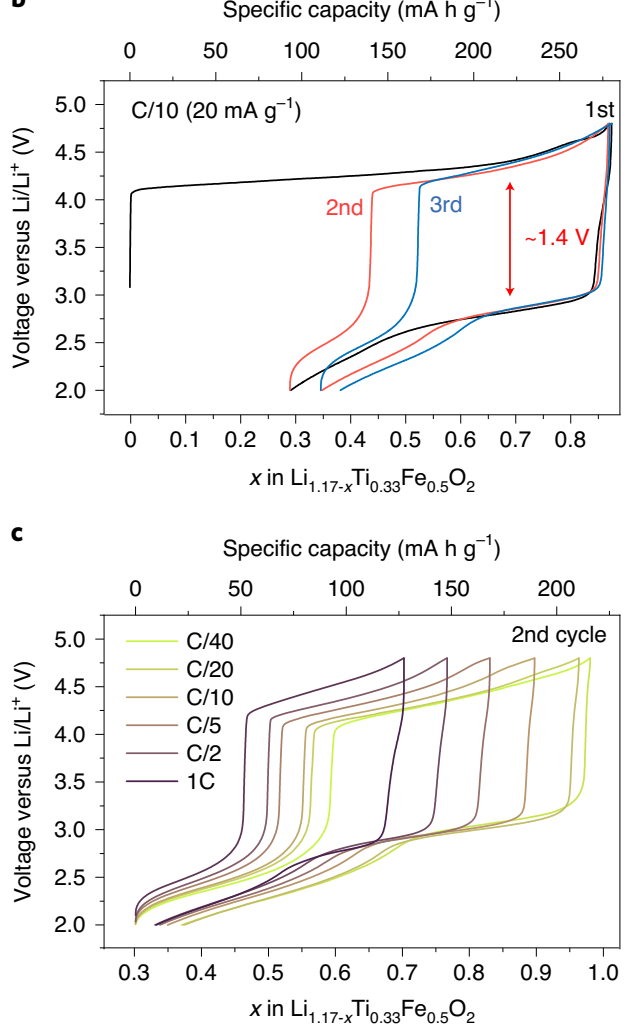

Fig. 1 | Structure and electrochemistry of LTFO compound. a, Rietveld refinement of the SXRD pattern (wavelength $\lambda=0.4579 \AA$ ) of the LTFO compound, with the structural parameters noted. The inset shows the crystal structure of LTFO, where the colours of the atoms are coded correspondingly in the formula. a.u., arbitrary units. b. The electrochemical profiles for the first three cycles of LTFO at $C / 10$. The huge voltage hysteresis of $\sim 1.4 \mathrm{~V}$ at the 2 nd cycle is marked with a double-headed arrow. c, The 2nd cycling curve for LTFO at different $\mathrm{C}$ rates.

the in situ XRD reveals a single-phase (solid-solution) behaviour, with the average structure maintaining the rock-salt disordered cubic structure during cycling. However, our first trials to fit the ex situ SXRD pattern of the sample charged to $4.8 \mathrm{~V}$ based on a regular disordered rock-salt structure could not take into account the $(002) /(220)$ relative intensity mismatch (Supplementary Fig. 7a). The refinement could be successfully improved by moving some TM ions from the octahedral $4 \mathrm{a}$ site to the interstitial tetrahedral $8 \mathrm{c}$ site of the rock-salt structure (Supplementary Fig. 7b), implying cation migration on charging. Neutron powder diffraction (NPD) confirms this cation migration and indicates that only Fe migrates (Fig. 2a,b and Supplementary Table 2), as schematically shown by 
a
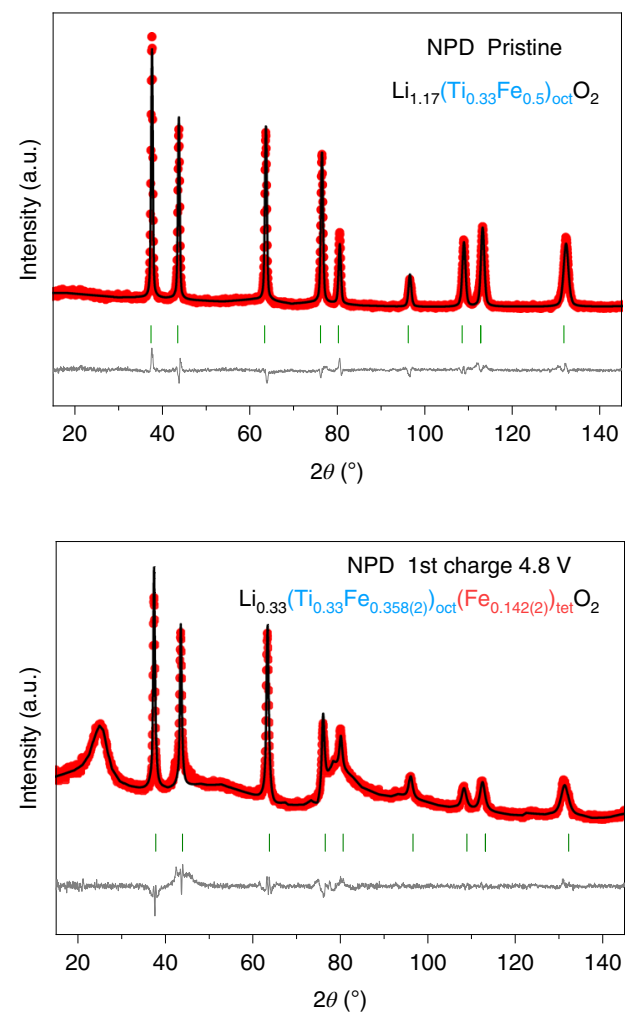

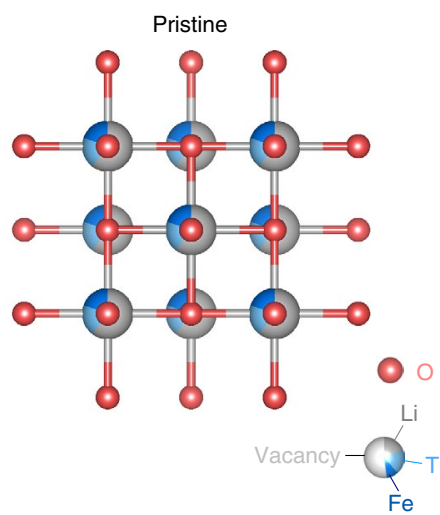

d

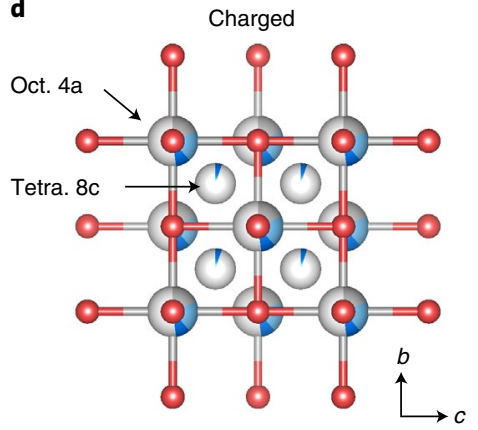

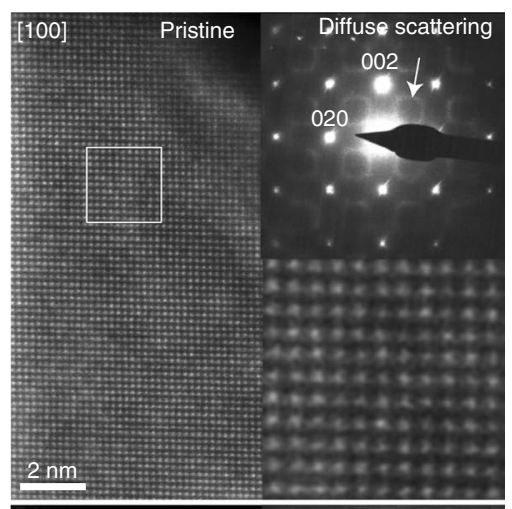
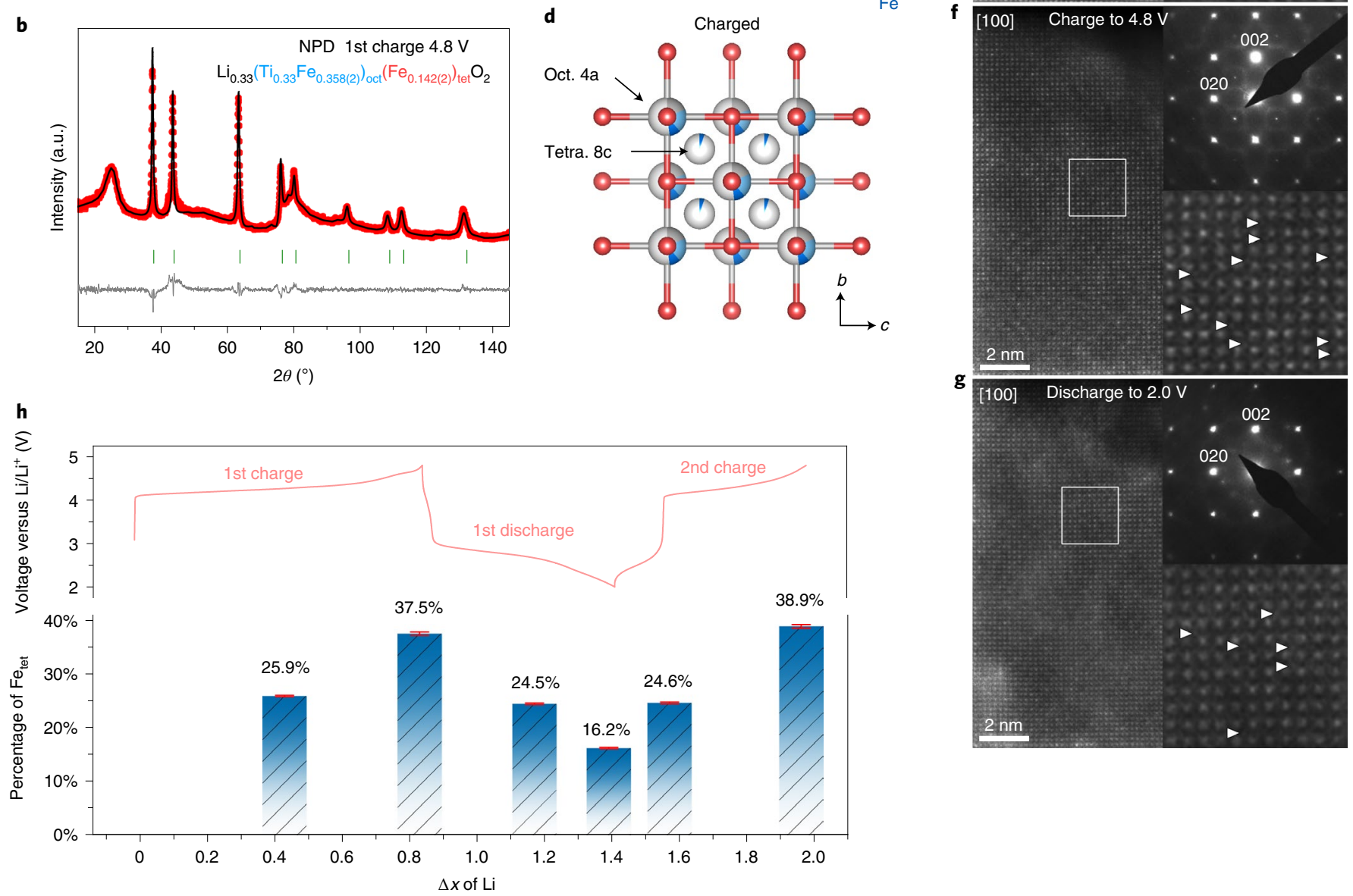

Fig. 2 | Reversible Fe migration. a,b, Rietveld refinement of the NPD pattern $(\lambda=1.5481 \AA$ ) of pristine LTFO (a) and the sample charged to $4.8 \mathrm{~V}$ (b). The compositions of these two samples are indicated by the chemical formulae, where the subscript 'oct' denotes an octahedral site and 'tet' denotes a tetrahedral site. The numbers in the brackets indicate the standard deviations of Fe content in different sites. The structure of the charged sample was finally solved with combined Rietveld refinement from NPD and SXRD data (Supplementary Table 2b). a.u., arbitrary units. c,d, Corresponding crystal structures of pristine (c) and charged LTFO (d). e-g, [100] HAADF-STEM images and ED patterns of pristine (e), charged to $4.8 \mathrm{~V}(\mathbf{f})$ and discharged to $2.0 \mathrm{~V}(\mathbf{g})$ samples. Magnified areas outlined with a white square are given in the right-bottom where the columns of tetrahedral voids occupied with

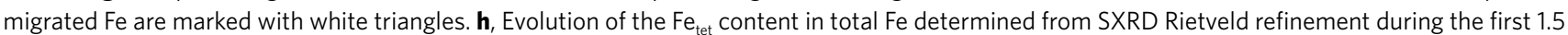
cycles, with the aligned electrochemical curve shown above. The red error bars at the top of every pillar show the standard deviations obtained from the refinement.

the structural change from Fig. 2c (pristine) to Fig. 2d (migrated). The migration of Fe to the tetrahedral site was also visualized by high angle annular dark-field scanning transmission electron microscopy (HAADF-STEM). As shown in Fig. 2e, the HAADF-STEM image along the [100] direction of the pristine LTFO demonstrates no TM cations occupying the tetrahedral voids, as no HAADF intensity is found at the centres of the squared bright dots which mark the octahedrally coordinated TM cationic positions (denoted as $\mathrm{TM}_{\text {oct. }}$ ). After charging (Fig. 2f), corresponding faint contrast can be recognized in the HAADF-STEM image (marked by white triangles), indicating the existence of tetrahedral $\mathrm{Fe}\left(\mathrm{Fe}_{\text {tet. }}\right)$. Also, the appearance of the $\mathrm{Fe}_{\mathrm{tet}}$ is accompanied by an extra ordering violating the parent face-centered unit cell as evidenced by sharp spots in the [100] ED pattern of the charged material (Fig. 2f). 
The reversibility of this migration behaviour was checked at various states of charge (SoC) through combined SXRD/NPD refinements (Supplementary Fig. 8 and Supplementary Tables 1 and 2). The evolution of the $\mathrm{Fe}_{\text {tet }}$ content along with the cycling state is plotted as a histogram in Fig. 2h. The results show that $37.5 \%$ of Fe moves to the tetrahedral sites on first charge and $21 \%$ moves back to the octahedral sites on the subsequent discharge, as further evidenced by the HAADF-STEM image (Fig. 2g). Regardless of the first charge, a $\sim 21 \%$ highly reversible $\mathrm{Fe}_{\text {oct }} \leftrightarrow \mathrm{Fe}_{\text {tet }}$ migration can be clearly observed from the 1st discharge to the 2nd charge process. This is supported by density functional theory (DFT) calculations (Supplementary Note 1) showing low kinetic barriers for $\mathrm{Fe}$ migration with $\mathrm{Fe}_{\text {tet }}$ sites that become as more thermodynamically favoured as the number of $\mathrm{Li}$ vacancies around the tetrahedral sites increases. Other than the reversible Fe migration, an irreversible local Li-TM ordering was also spotted in the HAADF-STEM images and this is discussed in Supplementary Fig. 2.

Although tempting, the scenario that Fe migration is linked to the large voltage hysteresis observed in LTFO is very unlikely. First, much less hysteresis is observed for the low-voltage pseudo-plateau below $2.7 \mathrm{~V}$ where reversible Fe migration also appears. Second, the path independence referred to above in LTFO suggests the hysteresis is independent of voltage window, that is, the amount of Fe migration. Therefore, an alternative explanation is needed.

Redox mechanism. Ex situ X-ray adsorption spectroscopy (XAS) and hard X-ray photoelectron spectroscopy (HAXPES) demonstrate the redox inactivity of $\mathrm{Ti}$, that is, it always stays as $\mathrm{Ti}^{4+}$ (Supplementary Fig. 9). In addition, in situ ${ }^{57} \mathrm{Fe}$ Mössbauer spectroscopy was performed with the pure ${ }^{57} \mathrm{Fe}$ LTFO sample and this was interpreted using three components from principal component analysis (PCA) associated with several Fe species, as indicated in Fig. $3 a-c$. Accessing accurately the Fe state is complex since, for instance, the $\mathrm{Fe}^{4+}{ }_{-} \mathrm{B}$ signal, corresponding to $\mathrm{Fe}^{4+}$ in octahedral sites (Fig. 3b), can equivalently be considered as $\mathrm{Fe}^{3+}$ in tetrahedral sites due to their similar isomer shifts ${ }^{23}$. However, we confidently assign this $\mathrm{Fe}$ signal to be $\mathrm{Fe}^{4+}$ in octahedral sites, combining the results of an in situ relaxation test as discussed in detail in Supplementary Fig. 10.

The evolution of these three components together with the different-valence Fe species along cycling is shown in Fig. 3d-g. The first charge demonstrates two distinct Fe redox processes, corresponding to the $\mathrm{Fe}^{3+} / \mathrm{Fe}^{4+}$ oxidation and the reduction of $\mathrm{Fe}^{4+}$ back to $\mathrm{Fe}^{3+}$ (see $\mathrm{Fe}^{4+}$ species in Fig. 3f), consistent with the two-stage process identified by in situ XRD and GITT data (Supplementary Fig. 6). The Fe valence change on first charge (from 3 to 3.4, Fig. 3g) accounts for only $\sim 0.2 \mathrm{Li}^{+}$(that is $0.5 \times 0.4$ ) of the overall charge capacity $\left(\sim 1 \mathrm{Li}^{+}\right)$. This implies a predominance of the $\mathrm{O}$ redox process that is validated by ex situ O $1 s$ HAXPES (\#1 $\rightarrow \# 3$ Fig. $3 i)$. Lastly, the reduction of $\mathrm{Fe}^{4+}$ (Fig. 3f, $\mathrm{Fe}^{4+}$ species) towards the end of the charge is consistent with a reductive coupling mechanism ${ }^{5,24}$, similar to previous findings in $\mathrm{Li}_{4} \mathrm{FeSbO}_{6}$ (ref. ${ }^{25}$ ). Turning to the first discharge plateau at $\sim 2.8 \mathrm{~V}(\# 3 \rightarrow \# 4)$, the remaining amount of $\mathrm{Fe}^{4+}$ is reduced back to $\mathrm{Fe}^{3+}$ (Fig. $3 \mathrm{~g}$ ) while $\mathrm{O}$ is largely reduced, indicative of a partially reversible $\mathrm{O}^{2-} / \mathrm{O}^{(2-n)-}$ redox chemistry $(\# 3 \rightarrow \# 4$ in Fig. 3i). On further reduction $\mathrm{Fe}^{3+}$ partially converts to $\mathrm{Fe}^{2+}$ (Fig. 3f, $\mathrm{Fe}^{2+}$ species) with little oxygen contribution (\#4 $\rightarrow \# 5$ in Fig. $3 \mathrm{i}$ ). In the second charge, $\mathrm{Fe}^{2+}$ is reversibly oxidized to $\mathrm{Fe}^{3+}$ below $4 \mathrm{~V}$ and $\mathrm{Fe}^{4+}$ species appear again but in a lesser amount at the high voltage plateau above $4 \mathrm{~V}$, accompanied by a highly reversible contribution of oxygen redox (\#6 $\rightarrow \# 7$ in Fig. 3i). It is worth mentioning that no trace of $\mathrm{Fe}^{4+}$ could be detected by ex situ Fe $2 p$ HAXPES (Fig. 3h) or ex situ Fe K-edge XAS (Supplementary Fig. 9c) recorded on the first 1.5 cycles, suggesting that $\mathrm{Fe}^{4+}$ might be a long-living non-equilibrium state.
GITT analysis. GITT measurements from 1st discharge to 2nd charge performed at both room temperature $\left(25^{\circ} \mathrm{C}\right)$ and $55^{\circ} \mathrm{C}$ confirm this hypothesis (Fig. 4). The GITT profile at $55^{\circ} \mathrm{C}$ shows nearly no gap between charge and discharge for both the cationic and anionic redox processes (Fig. 4b), while at room temperature solely the $\mathrm{Fe}^{3+} / \mathrm{Fe}^{2+}$ redox reaches equilibrium (Fig. 4a). Similar results were also obtained for the 8th cycle (Supplementary Fig. 11) except for some capacity fading. Long-time relaxations within the oxygen redox range at $25^{\circ} \mathrm{C}$ show a prompt recovery of the voltage in discharge (lower inset in Fig. 4c), while it takes more than 60 hours for the potential to reach equilibrium in charge (upper inset in Fig. 4c). Moreover, this relaxation proceeds by a two-stage behaviour (upper inset in Fig. 4c), which could result from two different relaxation processes that are coupled together. At $55^{\circ} \mathrm{C}$ (Fig. $4 \mathrm{~d}$ inset) the relaxation nearly replicated the $25^{\circ} \mathrm{C}$ voltage-drop profile but over a threefold shorter time. Additionally, we also measured the capacity of two cells cycled with (red solid curves) and without (black dashed curves) relaxations and found that they are roughly identical at both 25 and $55^{\circ} \mathrm{C}$, as shown in Fig. $4 \mathrm{c}$, d, respectively. This provides confidence that the measured voltage decay as a function of resting time is not due to self-discharge phenomena that are usually enhanced at high voltages and increased temperature.

Altogether, GITT analysis shows that the thermodynamic pathway between charge and discharge is a temperature-activated process. This calls for reconsidering the whole picture regarding the thermodynamics and kinetics of voltage hysteresis. The overpotential of any specific electrode enlists several components associated with various electronic/atomic motions having different time scales that are related to different kinetic barriers, such as Ohmic resistance (IR drop) and Li diffusion (Supplementary Fig. 12). From GITT (Fig. 4a) the cumulated IR drop and Li diffusion account for a $\sim 0.3 \mathrm{~V}$ polarization for the $\mathrm{Fe}^{3+} / \mathrm{Fe}^{2+}$ redox process at $\sim 2.5 \mathrm{~V}$. By contrast, through the oxygen redox region at higher potential, once the cumulated IR drop and Li diffusion overpotential are subtracted, there is still a $\sim 1 \mathrm{~V}$ OCV hysteresis, mainly nested in the charge process, that cannot be readily eliminated by short-period GITT at $25^{\circ} \mathrm{C}$ (Fig. 4a). The origin of this hysteresis will be revealed later.

In situ characterizations of relaxation. The electronic and structural evolution of the material along the relaxation process was examined by in situ techniques, starting at the mid-point of the second charge (O-redox plateau), as shown in Fig. 5. To avoid the self-discharge problem, Mössbauer spectra were collected on relaxing a dried electrode in a dry cell. The electrode was rapidly recovered from a cell charged to the targeted voltage, and then washed with dimethyl carbonate (DMC) and dried at reduced pressure. The data reveal the progressive reduction of $\mathrm{Fe}^{4+}$ as a function of relaxation time (Fig. 5a), which is even faster when relaxation is started from the middle of the first charge (Supplementary Figs. 10 and 13). In the absence of self-discharge the most feasible explanation to account for this reduction of $\mathrm{Fe}^{4+}$ is a charge redistribution between $\mathrm{Fe}^{4+}$ and $\mathrm{O}^{2-}$ through an internal redox process, leading to the thermodynamic equilibrium state $\mathrm{Fe}^{3+}-\mathrm{O}^{(2-n)-} / \mathrm{O}_{2}$ (molecule). This confirms our ex situ HAXPES and Mössbauer spectroscopy data.

To interrogate how such an internal redox process could affect local structure during relaxation we resorted to in situ XAS and XRD experiments. In situ extended X-ray absorption fine structure (EXAFS) data show little change around Ti (Fig. 5b) during relaxation. By contrast, in situ Fe K-edge EXAFS data reveal a noticeable intensity damping of the $\mathrm{Fe}-\mathrm{M}(\mathrm{M}=\mathrm{Fe} / \mathrm{Ti})$ coordination shell (Fig. 5c), probably due to the $\mathrm{Fe}^{4+}-\mathrm{O}^{2-}$ charge redistribution. EXAFS fittings demonstrate that this change could be interpreted as a local structural variation of the $\mathrm{Fe}-\mathrm{M}$ shell as supported by the change of Debye-Waller factors and bond length of different scattering paths (Fig. $5 \mathrm{~d}$ and Supplementary Table 3 ). Moreover, in situ $\mathrm{XRD}$ collected on relaxation highlights a robust tiny shift of the 


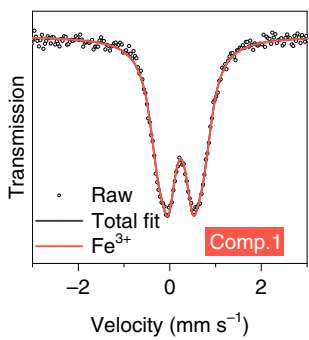

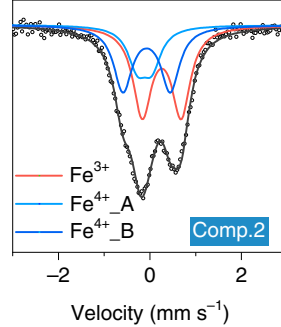

c

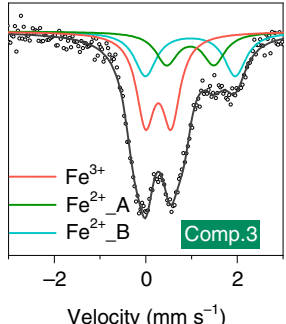

Velocity $\left(\mathrm{mm} \mathrm{s}^{-1}\right)$

d

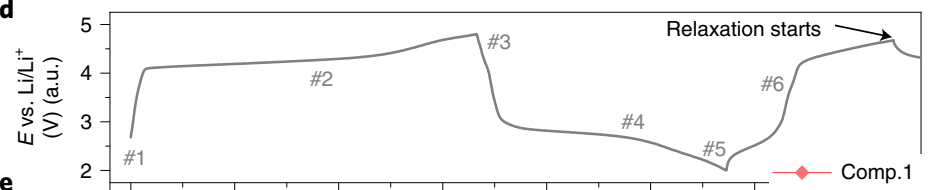

e
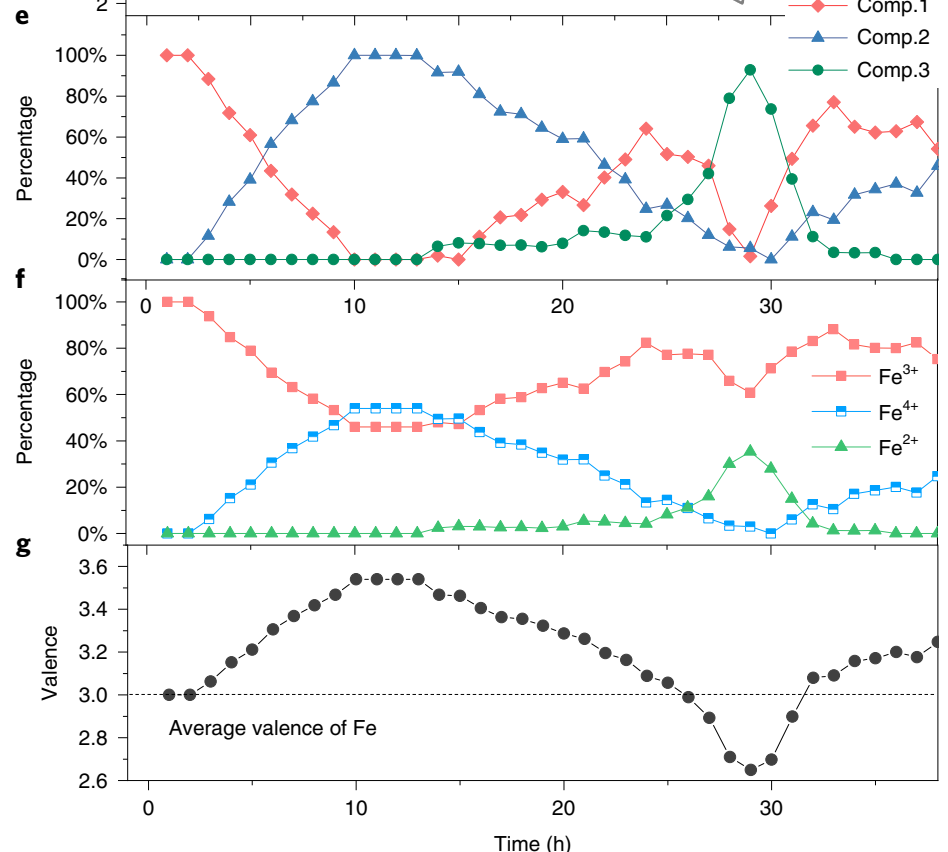

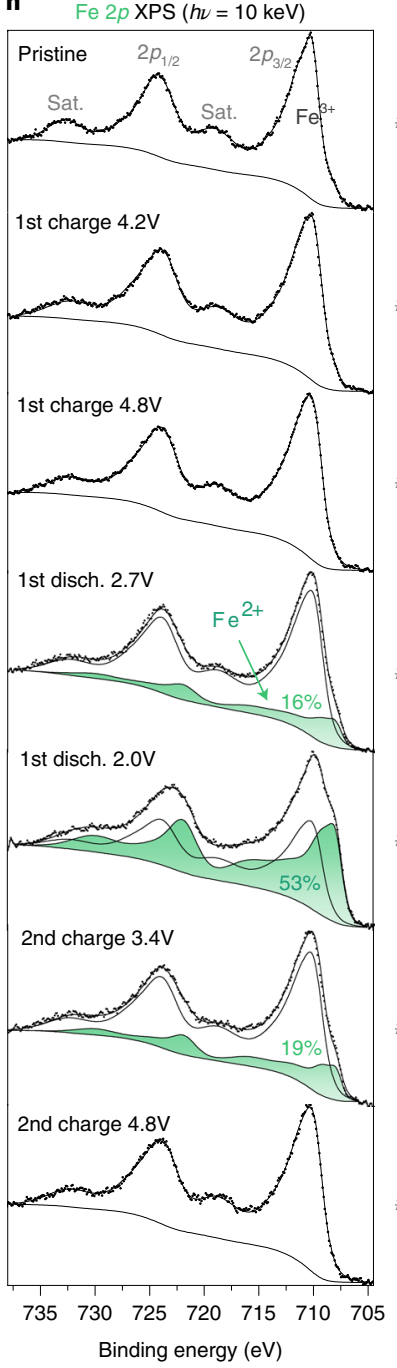

i

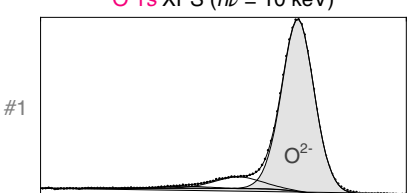

3
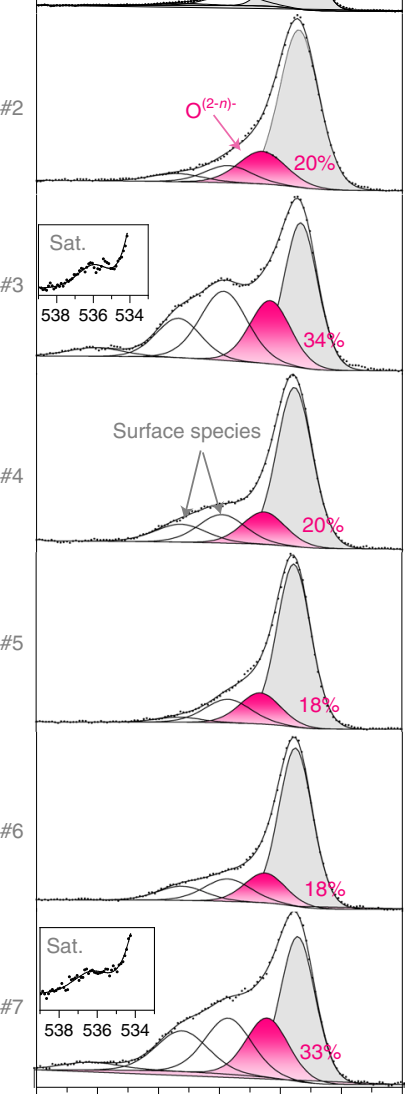

$\begin{array}{lllllll}538 & 536 & 534 & 532 & 530 & 528 & 526\end{array}$

Binding energy $(\mathrm{eV})$

Fig. 3 | Redox mechanism characterized by in situ ${ }^{57} \mathrm{Fe}$ Mössbauer spectroscopy and ex situ HAXPES. a-c, Representative fittings of ${ }^{57} \mathrm{Fe}$ Mössbauer spectra with the three components of the PCA: pristine (component 1) (a), intermediate (component 2) (b) and fully discharged (component 3) (c). The fittings enlisted several $\mathrm{Fe}^{3+}, \mathrm{Fe}^{4+}$ and $\mathrm{Fe}^{2+}$ species as indicated in the figures and in Supplementary Table 4. For $\mathrm{Fe}^{4+}$ or $\mathrm{Fe}^{2+}$, $\mathrm{A}$ and $\mathrm{B}$ denote different environments. All the Fe species are in octahedral sites unless specified additionally. d, Electrochemical curve of LTFO under in situ Mössbauer measurement. The cell was stopped at the 2 nd mid-charge to perform a relaxation test, as will be shown later. e, Evolution of the content of reconstructed components as a percentage during cycling. $\mathbf{f}, \mathbf{g}$, Evolution of different Fe species (f) and the average Fe valence (g). $\mathbf{h}$, Ex situ Fe $2 p$ HAXPES results with a photon energy of $h \nu=10 \mathrm{keV}$ (probe depth $\approx 39 \mathrm{~nm}$, bulk sensitive in this case) from pristine to 2 nd charge to $4.8 \mathrm{~V}$. The Fe $2 p$ spectra have two main peaks (as labelled by $2 p_{1 / 2}$ and $2 p_{3 / 2}$ ) due to spin-orbit splitting, with corresponding satellite peaks marked as 'Sat.'. The deconvolution of the spectra indicates the appearance of $\mathrm{Fe}^{2+}$ species (gradient green area) during the low-voltage plateau in addition to the $\mathrm{Fe}^{3+}$ species, with the percentage of $\mathrm{Fe}^{2+}$ noted alongside the peaks. $\mathbf{i}$, Ex situ O 1s HAXPES results from pristine to 2 nd charge to $4.8 \mathrm{~V}$. Three kinds of species were derived by deconvolution: (1) the lattice $\mathrm{O}^{2-}$ at $\sim 529.5 \mathrm{eV}$ (filled with grey); (2) oxidized lattice oxygen species $\mathrm{O}^{(2-n)-}(0<n<2)$ at around $530.5 \mathrm{eV}$ (filled with gradient pink), with its content denoted as a percentage near the peak; (3) the surface deposited species such as carbonates and so on. A satellite peak also repeatedly emerges when charged to 4.8 V (zoomed view labelled with 'Sat.'). The Fe $2 p$ and O 1s HAXPES spectra are all coded with numbers (\#1-\#6 except \#7 which is the end of the second charge) for tracking their SoC along the cycling curve in $\mathbf{d}$.

(002) peak accompanied by its narrowing ( Fig. 5e,f) that is more pronounced during the first relaxation plateau (from pattern \#1 to pattern \#2). This experimentally observed XRD peak sharpening indicates a micro-strain release which can be triggered by lithium diffusion during the internal redox process. Eventually, the relative constancy of (002) and (022) peak intensities (Supplementary Fig. 14) during relaxation implies the absence of $3 d$-metal cation migration, consistent with the barely changeable Fe-O shell peak of Fe K-edge EXAFS. This suggests that Fe migration is so fast that it cannot be captured by any in situ characterizations during relaxation, in line with the small activation energies computed with DFT (Supplementary Note 1). Therefore, we believe that Fe migration is not responsible for driving the relaxation that eliminates voltage hysteresis.

Correlating $\mathrm{O} 2 p-\mathrm{Fe} 3 d$ charge transfer with voltage hysteresis. The charge redistribution of $\mathrm{Fe}^{4+}-\mathrm{O}^{2-}$ along with relaxation is consistent with our inability to detect $\mathrm{Fe}^{4+}$ species by ex situ XAS and HAXPES, as the high-energy and high-flux X-ray beam may facilitate a rapid relaxation. This is an indication in favour of $\mathrm{Fe}^{4+}$ as a 

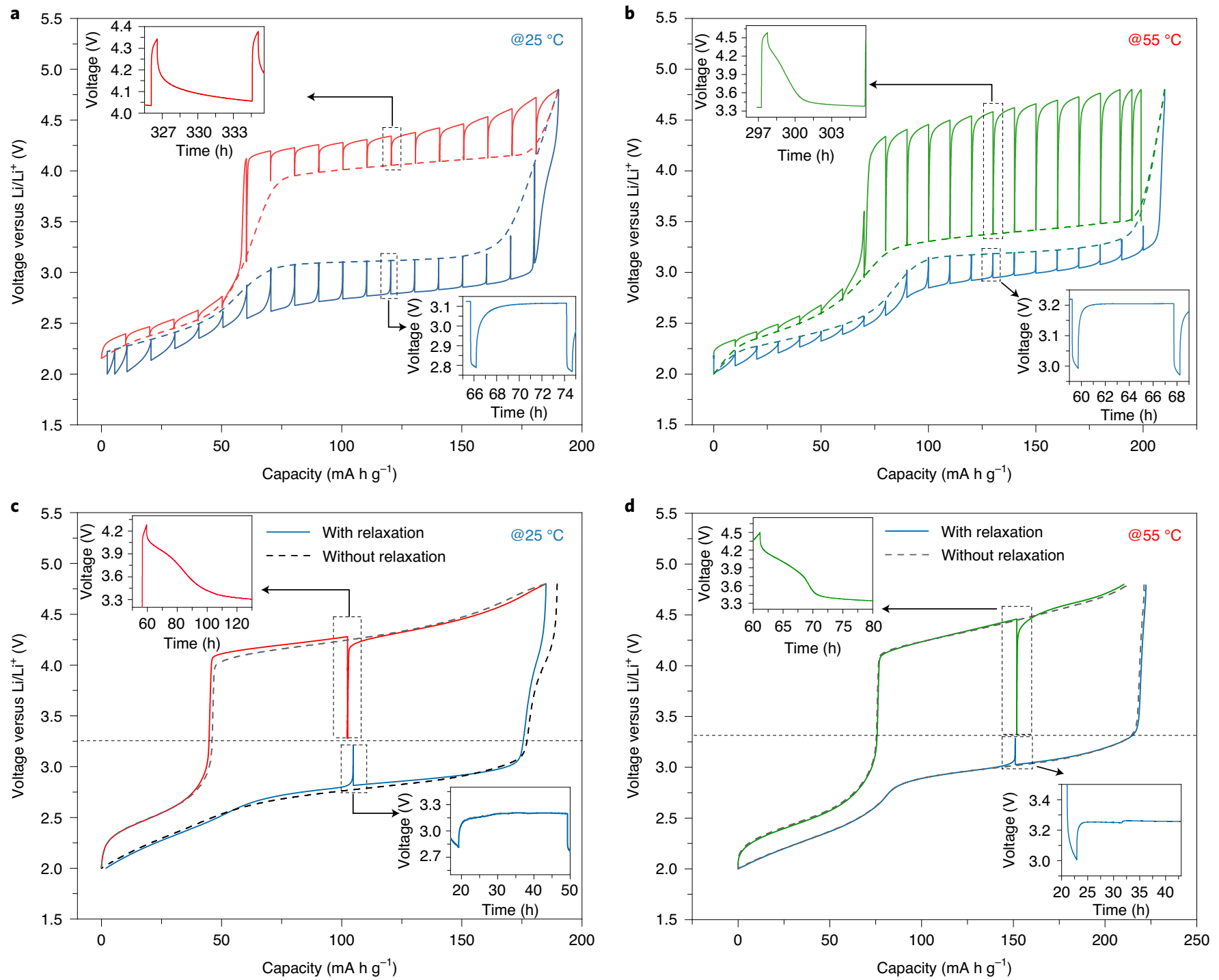

Fig. 4 | GITT analysis of LTFO from the 1st discharge to the 2 nd charge process. a,b, GITT results at 25 and $55^{\circ} \mathrm{C}$. The tests were performed with a $0.5 \mathrm{~h}$ pulse at $\mathrm{C} / 10$ followed by $8 \mathrm{~h}$ relaxation every step. The representative relaxation processes for charge and discharge are snapshotted in the insets. The OCV points outline the quasi-static charge-discharge paths (dashed curves). Specifically, during the oxygen-redox region, the relaxation during charge seemingly never ends with the potential always drifting, while at discharge it stabilizes rapidly (insets in Fig. 4a), suggesting asymmetrical kinetics between charge and discharge. $\mathbf{c , d}$, Long-time relaxation tests at $25(\mathbf{c})$ and $55^{\circ} \mathrm{C}(\mathbf{d})$. The cycling rate is $\mathrm{C} / 10$. Every relaxation process was carried out until the equilibrium potential (marked by straight dashed lines) was reached, with the potential-drifting profiles versus time shown in the insets. The electrochemical curves with relaxation (solid) and without relaxation (dashed) are compared.

non-equilibrium state and consequently a supplementary argument for ascribing its return to $\mathrm{Fe}^{3+}$ via a sluggish process that is likely to be associated with significant reorganization energy that could be at the origin of the large voltage hysteresis.

In line with Marcus theory ${ }^{26-28}$ (a brief introduction can be found in Supplementary Note 2), the anionic redox process taking place during charging in the LTFO electrode is a typical non-adiabatic process due to the lack of orbital overlapping between $\mathrm{O}(2 p)$ lone-pair states and $\operatorname{TM}(n d)$ states. As pictured in Fig. 6a, the oxidation of the $\mathrm{Fe}^{3+} / \mathrm{O}^{2-}$ pristine state (grey parabola) to the charged $\mathrm{Fe}^{3+} / \mathrm{O}^{-}$state (red parabola) does not follow a stepwise adiabatic reaction mechanism along one unique reaction coordinate. Instead, it undergoes a concerted mechanism involving an intermediate metastable state $\left(\mathrm{Fe}^{4+} / \mathrm{O}^{2-}\right.$, blue parabola), the potential energy surface (PES) of which is coupled with the pristine and $\mathrm{Fe}^{3+} / \mathrm{O}^{-}$equilibrium states through a different reaction coordinate associated with reasonable kinetic barriers. In simple terms, to overcome the large kinetic barrier associated with the reorganization of the oxygen network required to directly transform the $\mathrm{Fe}^{3+} / \mathrm{O}^{2-}$ pristine state into the charged $\mathrm{Fe}^{3+} / \mathrm{O}^{-}$equilibrium state, the system follows an adiabatic pathway through the $\mathrm{Fe}^{4+} / \mathrm{O}^{2-}$ intermediate state. Then, it evolves along a new reaction coordinate (Fig. 6a) which is levered by the $\mathrm{Fe}^{4+} \mathrm{Jahn}-\mathrm{Teller}$ distortion and whose rotation with respect to the initial coordinate enables the system to easily de-excite into the $\mathrm{Fe}^{3+} / \mathrm{O}^{-}$ground state (equilibrium) to lower its energy with the appropriate local geometry (distortion of the O network).

Such a concerted mechanism is fully inferred from DFT calculations. As depicted in Fig. 7a the Fukui function computed for the pristine material shows that the early stage of charge involves $\sigma$-type $\mathrm{Fe}(3 d)-\mathrm{O}(2 p)$ hybridized states, rather than the localized oxygen lone-pair states lying just below the Fermi level in the density of states (DOS) (Fig. $7 \mathrm{~b}$ and Supplementary Fig. 16). The facile 

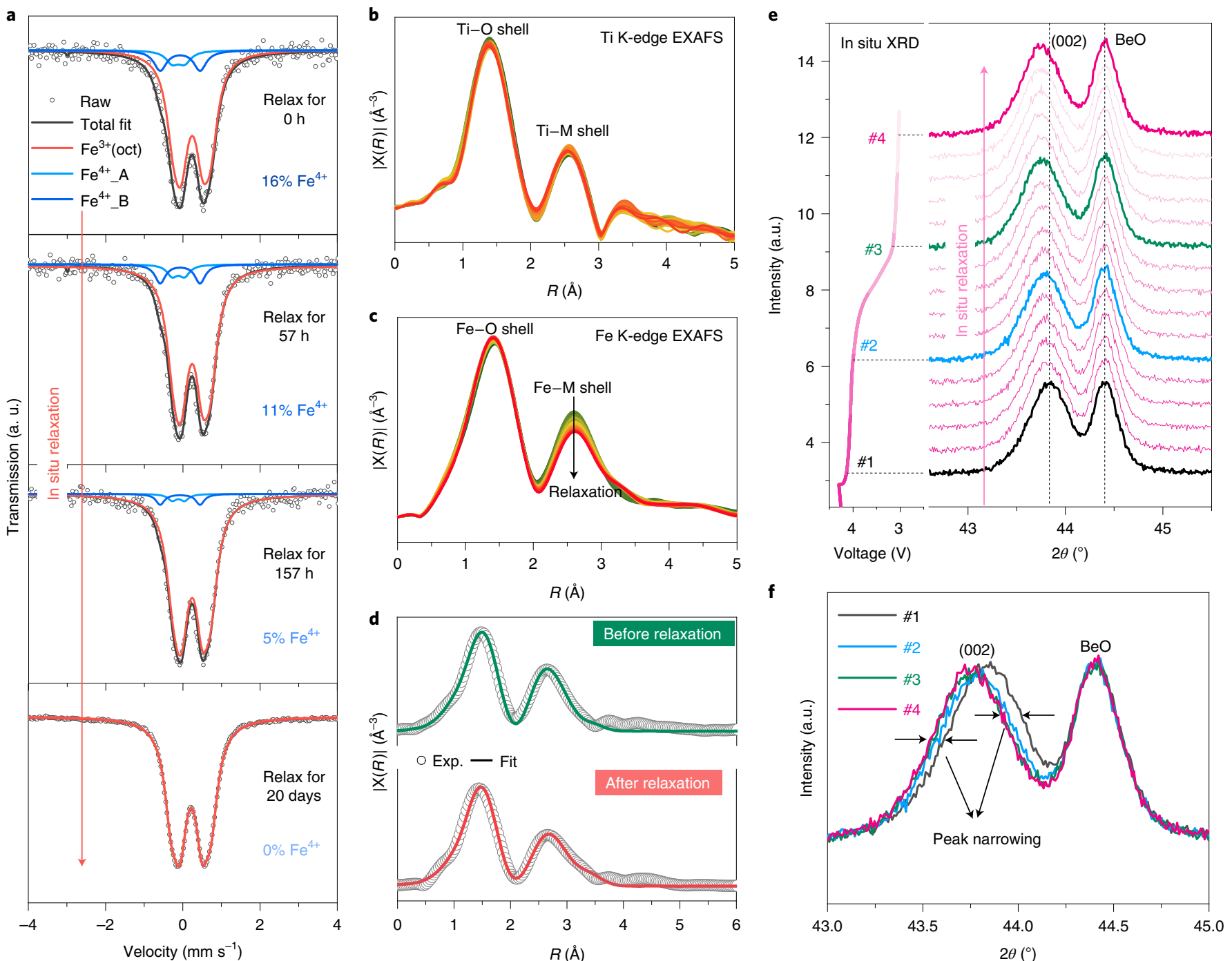

Fig. $\mathbf{5}$ | In situ characterizations of the electronic and geometric structural evolutions during relaxation. $\mathbf{a}$, The evolution of the in situ ${ }^{57} \mathrm{Fe} \mathrm{Mössbauer}$ spectra as a function of the relaxation time. The model used in the fitting is a combination of components 1 and 2 from PCA (details are shown in Supplementary Table 5), as the spectra can be fully reconstructed by these two components (see component evolution in Fig. 3e). In the legends, $\mathrm{A}$ and $\mathrm{B}$ denote different environments of $\mathrm{Fe}^{4+}$. a.u., arbitrary units. b.c, Fourier-transformed $k^{2}$-weighted EXAFS oscillations of in situ Ti (b) and $\mathrm{Fe}$ (c) $\mathrm{K}$-edge XAS spectra. The two peaks of the radial distribution functions correspond to Ti/Fe- $\mathrm{O}$ and $\mathrm{Ti} / \mathrm{Fe}-\mathrm{M}(\mathrm{M}=\mathrm{Ti}$, $\mathrm{Fe})$ shells, as noted in the figures. The obvious trend of the Fe-M shell along with relaxation is clearly visible as marked by the vertical arrow. d, EXAFS fittings of Fe before and after relaxation, with the fitting parameters shown in Supplementary Table 3. e, In situ XRD patterns during relaxation, with the voltage versus time curve shown on the left. In addition to the peak of LTFO (002), the peak at around $44.4^{\circ}$ is from $\mathrm{BeO}$, which is steady along relaxation and thus can be used as a reference peak. $\mathbf{f}$, The number-coded spectra (\#1 to \#4) are superimposed together to highlight the peak shape change.

contraction of the Fe-O bond (Supplementary Fig. 17) associated with this $\mathrm{Fe}$ oxidation process sets the reaction coordinate along which the system dynamically evolves. Increasing the number of holes in the $\sigma$-type $\mathrm{Fe}(3 d)-\mathrm{O}(2 p)$ states triggers the $\mathrm{Fe}^{4+}$ Jahn-Teller distortion which lowers the system symmetry, therefore allowing the system to evolve through a novel reaction coordinate which is compatible with the typical O-network distortion of anionic redox (Supplementary Fig. 17). Due to the new hybridization between the metal $d$ orbitals and the oxygen $2 p$ orbitals (originally the lone-pair states), an internal redox reaction occurs to lower the system energy and reach the thermodynamically stable $\mathrm{Fe}^{3+}-\mathrm{O}^{-}$state through sluggish structural reorganization. This equilibrium anion-oxidized state is experimentally confirmed by ex situ measurements evidencing only $\mathrm{Fe}^{3+}$ and theoretically proved by Bader charge (Fig. 7c) and DOS analysis as well as the calculated equilibrium potentials showing the sole oxidation of the anions (Supplementary Fig. 18). The charge redistribution of $\mathrm{Fe}^{4+}$ and $\mathrm{O}^{2-}$ also leads to another possible adiabatic reaction involving $\mathrm{O}_{2}$ molecules, in addition to reversible $\mathrm{O}$ redox, based on the calculated oxygen-release enthalpy as discussed in Supplementary Fig. 18d.

In light of these results, we propose the scenario pictured in Fig. $6 \mathrm{~b}$ to account for voltage hysteresis in LTFO. Within the $\mathrm{O}$ redox region, the charge/discharge follows a non-equilibrium path (blue curve) involving a ' $\mathrm{Fe}^{4+}-\mathrm{O}^{2-}$ intermediate state as a result of bypassing the direct electron removal from the localized $\mathrm{O}(2 p)$ lone-pair states. The system is then dynamically trapped in this ' $\mathrm{Fe}^{4+}-\mathrm{O}^{2-}$ ' intermediate until the rotation of the reaction coordinate allows its relaxation to the equilibrium $\mathrm{Fe}^{3+} / \mathrm{O}^{(2-n)-}$ ground state (red curve) via an internal $\mathrm{O} 2 p$ to $\mathrm{Fe} 3 d$ electron transfer. Moreover, in situ electrochemical impedance spectroscopy (EIS) study during 

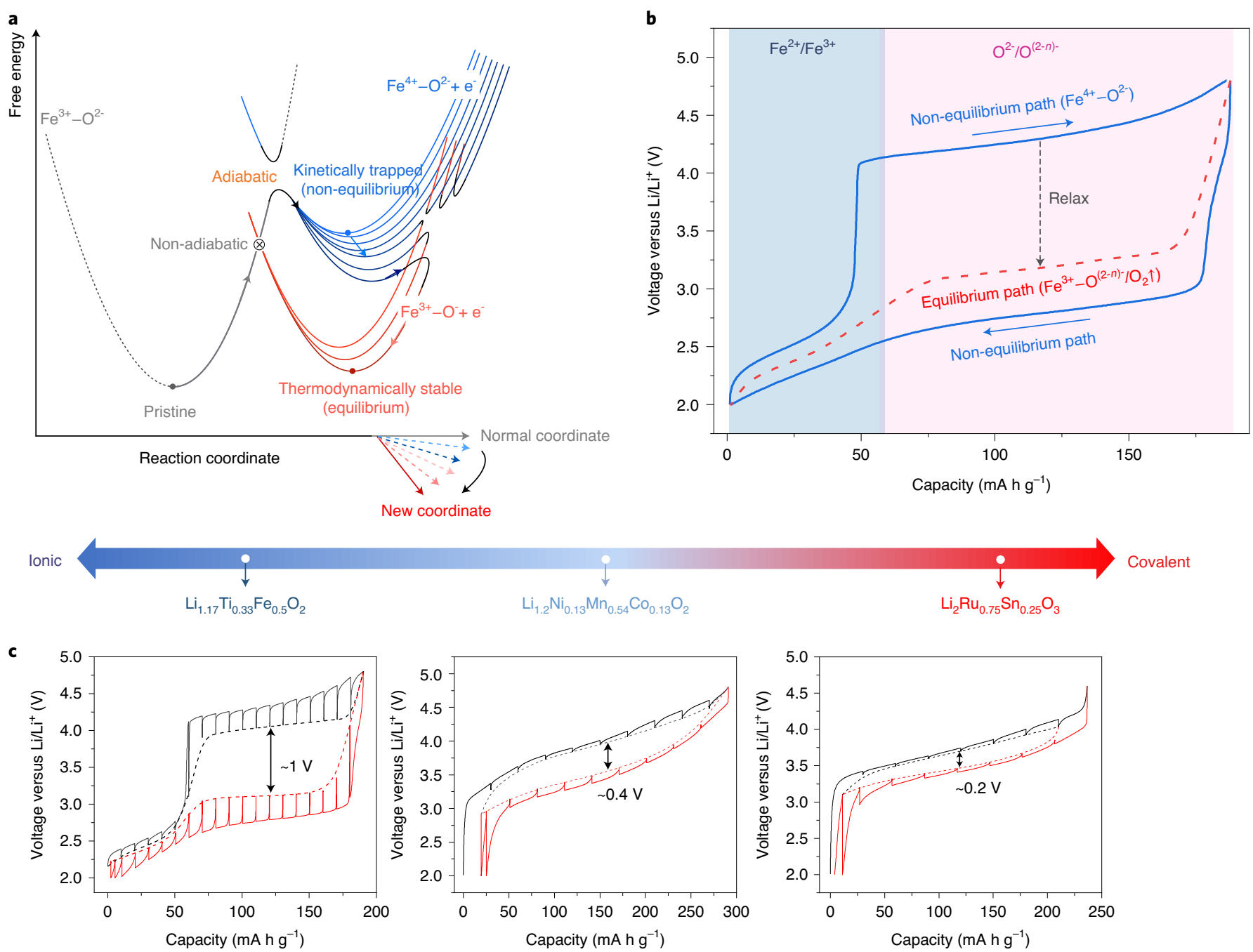

Fig. 6 | Kinetic and thermodynamic picture of voltage hysteresis. a, Scheme of the one-electron transfer reaction during $O$ redox based on Marcus theory. The grey, blue and red parabolas represent the PESs of pristine $\mathrm{Fe}^{3+-}-\mathrm{O}^{2-}$, ionized $\mathrm{Fe}^{4+}-\mathrm{O}^{2-}+\mathrm{e}^{-}$and $\mathrm{Fe}^{3+}-\mathrm{O}^{-}+\mathrm{e}^{-}$states, respectively. Here we have simplified the oxidized $\mathrm{O}$ species $\left(\mathrm{O}^{(2-n)}\right.$ - or $\mathrm{O}_{2}$ molecule) to be $\mathrm{O}^{-}$since it is a one-electron reaction. Note that indeed the PESs are all in 3D, but are plotted as $2 \mathrm{D}$ for clarity. The cross point ' $\otimes$ ' between grey and red parabola means these two PESs are not coupled along the reaction coordinate so that the system is unable to evolve adiabatically to the red PES. By contrast, the grey and blue PESs are coupled along the reaction coordinate which lifts the degeneracy at their crossing point, therefore allowing the system to follow an adiabatic path towards the blue PES. Once in the $\mathrm{Fe}^{4+-} \mathrm{O}^{2-}$ state, the system slowly evolves along a new reaction coordinate to lower its energy and de-excite into the $\mathrm{Fe}^{3+}-\mathrm{O}^{-}$ground state through $\mathrm{O}(2 p)-\mathrm{Fe}(3 d)$ charge transfer. The dynamical pathway of the electron transfer reaction is highlighted by the arrows attached to the PESs. b. Schematic summary of the voltage hysteresis in LTFO. The Fe ${ }^{3+} / \mathrm{Fe}^{2+}$ and $\mathrm{O}$ redox regions are highlighted with different colours. The charge and discharge follow a non-equilibrium path (blue solid curve) with $\mathrm{Fe}^{4+}-\mathrm{O}^{2-}$ while the equilibrium path (red dashed curve) comprises $\mathrm{Fe}^{3+-} \mathrm{O}^{(2-n)-} / \mathrm{O}_{2} \uparrow$. Note that here the non-equilibrium $\mathrm{Fe}^{4+-} \mathrm{O}^{2-}$ just statistically exists and is only a small portion of the total $\mathrm{Fe}-\mathrm{O}$ species. The black arrow indicates how the relaxation will drive the non-equilibrium state to the equilibrium state.

c, Voltage-hysteresis magnitude increases with the TM-O system ranging from a covalent system to an ionic system, as benchmarked by the three typical Li-rich compounds representing covalent, medium and ionic systems.

relaxation (Supplementary Fig. 19) proves that the non-equilibrium $\mathrm{Fe}^{4+}-\mathrm{O}^{2-}$ state possesses better charge-transfer kinetics than the equilibrium $\mathrm{Fe}^{3+}-\mathrm{O}^{-}$state as deduced by the hugely increased charge-transfer resistance after relaxation. This explains why the non-equilibrium path with $\mathrm{Fe}^{4+}$ is kinetically favoured. The sluggish kinetics of $\mathrm{O}$ redox are further supported by sluggish Li diffusion kinetics quantified from GITT (Supplementary Fig. 20) and the electrochemical quantitative analysis based on the phenomenological Butler-Volmer model (Supplementary Note 3). The analysis gives a much lower exchange current density of $(9.62 \pm 1.64) \times$ $10^{-7} \mathrm{~mA} \mathrm{~cm}^{-2}$ for the $\mathrm{O}$ redox reaction than normal cationic reactions (for example, $\mathrm{Fe}^{3+/ 2+}\left(0.17 \mathrm{~mA} \mathrm{~cm}^{-2}\right)$ in $\left.\mathrm{LiFePO}_{4}\right)^{29}$.

\section{Discussion}

In classical insertion compounds, a long and commonly held belief consists of associating hysteresis with the substantial disorder or motion of cations within the host structures. This leads to the straightforward extrapolation that hysteresis in Li-rich compounds originates from the TM cation migration triggered by the anionic redox process. However, the relationship between cation migration and hysteresis is not universal and breaks down for compounds having voltage hysteresis without cation migration, such as $\mathrm{Na}_{2 / 3}\left[\mathrm{Mg}_{0.28} \mathrm{Mn}_{0.72}\right] \mathrm{O}_{2}$ (ref. ${ }^{30}$ ) and $\mathrm{Na}_{2} \mathrm{RuO}_{3}$ (ref. ${ }^{31}$ ). An additional counter-example is $\mathrm{NaCrSSe}$, showing negligible hysteresis but with substantial Cr migration ${ }^{32}$. Therefore, cation migration is not 

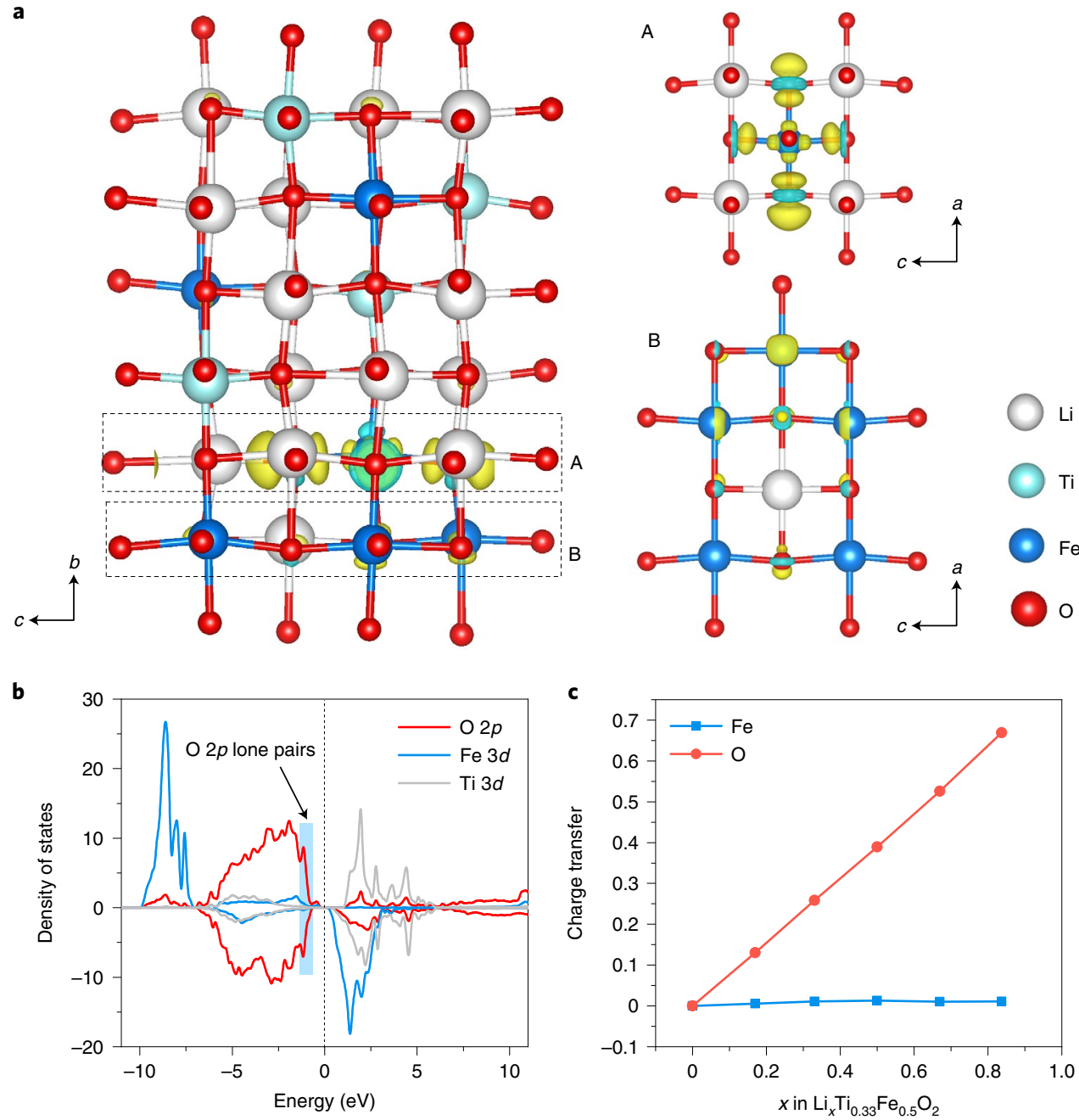

Fig. 7 | Theoretical calculations of the redox process of LTFO. a, Fukui function analysis of pristine LTFO by removing 0.2 electrons from the system (222 electrons). Local views from another direction are also provided as marked by A and B. The charge density change in yellow is positive (electron loss) and in cyan is negative (electron gain). The isosurface value is set as $7 \times 10^{-3}$. b. Projected DOS of pristine LTFO calculated using the DFT $+U$ framework. The $\mathrm{O}(2 p)$ states corresponding to $\mathrm{O}(2 p)$ lone pairs are highlighted. $\mathbf{c}$, Bader charge analysis of LTFO during its delithiation process.

necessarily associated with structural reorganizations triggered by the anionic redox process and should not be blamed for voltage hysteresis unless clear evidence is established. Huge voltage hysteresis is frequently found in the Li-rich cation-disordered rock-salt phases pioneered by the groups of Ceder and Yabuuchi ${ }^{33-36}$. This might lead to the belief that cation disorder may play a key role in hysteresis. However, large hysteresis exclusively appears in anionic redox process for cation-disorder compounds like LTFO. This is supported by the presence of minor hysteresis ${ }^{37}$ in fully disordered compounds, like $\mathrm{Li}_{2} \mathrm{VO}_{2} \mathrm{~F}$, having sole cationic redox $\left(\mathrm{V}^{3+} / \mathrm{V}^{5+}\right)$ and somewhat highlights the independence of voltage hysteresis from cation disorder. Lastly, a redox inversion model rooted in the sequential oxidation of $\mathrm{M}$ then $\mathrm{O}$ on charging and reduction of $\mathrm{M}$ then $\mathrm{O}$ on discharging (instead of $\mathrm{O}$ then $\mathrm{M}$ ) was also used to explain hysteresis in a few Li-rich rock-salt compounds $\left(\mathrm{Li}_{1.3} \mathrm{Ni}_{0.27} \mathrm{Ta}_{0.43} \mathrm{O}_{2}\right)^{2,17}$. Although elegant, this model, relying on thermodynamics rather than on kinetics, has limitations since it could not explain the hysteresis in some compounds, such as $\mathrm{Li}_{2} \mathrm{Ru}_{0.75} \mathrm{Sn}_{0.25} \mathrm{O}_{3}$ and LTFO, that are free of redox inversion.

By contrast, our explanation of the voltage hysteresis involving $\mathrm{O}(2 p)$-to-TM $(n d)$ charge transfer is of higher universality. $\mathrm{O}(2 p)$-to-TM $(n d)$ charge transfer is common to numerous Li-rich compounds in which the anionic redox process is described by a first-step cationic oxidation followed by a charge redistribution between $\mathrm{TM}$ and $\mathrm{O}$. A few examples are $\mathrm{Li}_{2} \mathrm{Ru}_{0.75} \mathrm{Sn}_{0.25} \mathrm{O}_{3}\left(\mathrm{Ru}^{6+}\right)^{5}$, $\mathrm{Li}_{2} \mathrm{Ir}_{y} \mathrm{Sn}_{1-y} \mathrm{O}_{3}\left(\mathrm{Ir}^{>5.5+}\right)^{7}$ and $\mathrm{Li}_{2} \mathrm{MnO}_{3}$-based phases $\left(\mathrm{Mn}^{7+}\right)^{38}$. Although this mechanism is named differently by authors (reductive coupling or ligand-to-metal charge transfer), it invariably assumes the existence of an intermediate cation-oxidized excited state that has, however, never been observed so far. This lack of evidence no longer exists owing to this unique LTFO system for which we could successfully capture the intermediate $\mathrm{Fe}^{4+}$ species as evidence of a reductive coupling mechanism $(\mathrm{O}(2 p)$-to-TM $(n d)$ charge transfer) for anionic redox.

Moreover, our study also provides a general understanding of the amplitude variation of voltage hysteresis in the large family of Li-rich rock-salt compounds. First, as a qualitative trend the voltage hysteresis magnitude is increased from covalent $\left(\mathrm{Li}_{2} \mathrm{Ru}_{0.75} \mathrm{Sn}_{0.25} \mathrm{O}_{3}\right)$ to medium covalent/ionic $\left(\mathrm{Li}_{1.2} \mathrm{Ni}_{0.13} \mathrm{Mn}_{0.54} \mathrm{Co}_{0.13} \mathrm{O}_{2}\right)$ and more pronounced ionic $\left(\mathrm{Li}_{1.17} \mathrm{Ti}_{0.33} \mathrm{Fe}_{0.5} \mathrm{O}_{2}\right.$ ) systems (Fig. 6c), regardless of the cation order/disorder (remembering that the local structure for LTFO is very similar to that in the layered oxides as seen from transmission electron microscopy (TEM) results). This can be interpreted as: the higher the covalence, the larger the electron 
delocalization over the $\mathrm{M}-\mathrm{O}$ bonds, leading to lower kinetic barriers for electron transfer that will result in smaller voltage hysteresis. This trend can also be extended to more covalent sulfides or even selenides, as supported by the much smaller hysteresis that they display $^{9,31,39}$. It is worth mentioning that disorder is not a prerequisite for large hysteresis since it is also present in cation-ordered $\mathrm{Li}_{4+x} \mathrm{Ni}_{1-x} \mathrm{WO}_{6}$ phases with $d^{0} \mathrm{~W}^{6+}$ cations ${ }^{16}$. It is also reported that F substitution could alleviate hysteresis in cation-disordered compounds although it increases the ionicity ${ }^{40,41}$. This does not break the covalence-ionicity trend in hysteresis either, as F substitution lowers the TM valence and so increases the cationic-redox contribution at the cost of anionic redox, therefore with less hysteresis. Additionally, huge hysteresis can also be found in $d^{10}$ metal/ metalloid-based compounds, like those of $d^{0}$-metals that show high ionicity such as the previously reported $\mathrm{Li}-\mathrm{Te}-\mathrm{Fe}-\mathrm{O}\left(\right.$ ref. ${ }^{42}$ ) and $\mathrm{Li}_{4} \mathrm{FeSbO}_{6}$ (ref. ${ }^{25}$ ) (Supplementary Table 7).

A burning question from a practical point of view is how to escape this hysteresis jail that plagues the use of anionic-redox materials. A first direction consists of increasing the $d-p$ hybridization through increasing covalence of the TM-O bonds or through less electronegative and softer anions such as $\mathrm{S}$ or Se. A second is to use the local structure for preventing ligand dimerization and by the same token local structural distortion, like what has been undertaken with $\mathrm{P} 2-\mathrm{Na}_{0.6}\left[\mathrm{Li}_{0.2} \mathrm{Mn}_{0.8}\right] \mathrm{O}_{2}$ (ref. ${ }^{19}$ ) and $\mathrm{Na}_{2} \mathrm{Mn}_{3} \mathrm{O}_{7}$ (ref. ${ }^{43}$ ) phases. Apparently, in such ribbon or mesh superstructures, oxygen redox could proceed by involving $\mathrm{O} 2 p$ localized holes or delocalized $\pi$ states $^{44}$ that are free of the reductive coupling process, like those having redox from 'TM-O' bonding states (for example $\mathrm{LiCoO}_{2}$ (ref. ${ }^{1}$ ) and $\mathrm{Na}_{\mathrm{x}}\left[\mathrm{Ni}_{1 / 3} \mathrm{Mn}_{2 / 3}\right] \mathrm{O}_{2}$ (ref. $\left.{ }^{45}\right)$ ) rather than lone-pair states. Therefore, we believe this fresh view of the hysteresis of Li-rich compounds has revealed the crucial importance of ligand-to-metal charge transfer in the anionic redox process, which should help chemically cure the energy efficiency roadblock penalizing anionic-redox based materials and thereby their implementation in real-world applications.

\section{Online content}

Any methods, additional references, Nature Research reporting summaries, source data, extended data, supplementary information, acknowledgements, peer review information; details of author contributions and competing interests; and statements of data and code availability are available at https://doi.org/10.1038/ s41557-021-00775-2.

Received: 27 November 2020; Accepted: 14 July 2021; Published online: 16 September 2021

\section{References}

1. Assat, G. \& Tarascon, J.-M. Fundamental understanding and practical challenges of anionic redox activity in Li-ion batteries. Nat. Energy 3, 373-386 (2018).

2. Ben Yahia, M., Vergnet, J., Saubanere, M. \& Doublet, M. L. Unified picture of anionic redox in Li/Na-ion batteries. Nat. Mater. 18, 496-502 (2019).

3. $\mathrm{Li}, \mathrm{M}$. et al. Cationic and anionic redox in lithium-ion based batteries. Chem. Soc. Rev. 49, 1688-1705 (2020).

4. Li, B. \& Xia, D. Anionic redox in rechargeable lithium batteries. Adv. Mater. 29, 1701054 (2017).

5. Sathiya, M. et al. Reversible anionic redox chemistry in high-capacity layered-oxide electrodes. Nat. Mater. 12, 827-835 (2013).

6. McCalla, E. et al. Visualization of $\mathrm{O}-\mathrm{O}$ peroxo-like dimers in high-capacity layered oxides for Li-ion batteries. Science 350, 1516-1521 (2015).

7. Hong, J. et al. Metal-oxygen decoordination stabilizes anion redox in Li-rich oxides. Nat. Mater. 18, 256-265 (2019).

8. Perez, A. J. et al. Approaching the limits of cationic and anionic electrochemical activity with the Li-rich layered rocksalt $\mathrm{Li}_{3} \mathrm{IrO}_{4}$. Nat. Energy 2, 954-962 (2017).

9. Saha, S. et al. Exploring the bottlenecks of anionic redox in Li-rich layered sulfides. Nat. Energy 4, 977-987 (2019).
10. Flamary-Mespoulie, F. et al. Lithium-rich layered titanium sulfides: cobalt- and nickel-free high capacity cathode materials for lithium-ion batteries. Energy Storage Mater. 26, 213-222 (2020).

11. Assat, G. et al. Fundamental interplay between anionic/cationic redox governing the kinetics and thermodynamics of lithium-rich cathodes. Nat. Commun. 8, 2219 (2017).

12. Gent, W. E. et al. Coupling between oxygen redox and cation migration explains unusual electrochemistry in lithium-rich layered oxides. Nat. Commun. 8, 2091 (2017).

13. Croy, J. R. et al. Examining hysteresis in composite $x \mathrm{Li}_{2} \mathrm{MnO}_{3} \cdot(1-x) \mathrm{LiMO}_{2}$ cathode structures. J. Phys. Chem. C 117, 6525-6536 (2013).

14. Dogan, F. et al. Re-entrant lithium local environments and defect driven electrochemistry of Li- and Mn-rich Li-ion battery cathodes. J. Am. Chem. Soc. 137, 2328-2335 (2015).

15. Eum, D. et al. Voltage decay and redox asymmetry mitigation by reversible cation migration in lithium-rich layered oxide electrodes. Nat. Mater. 19, 419-427 (2020)

16. Taylor, $\mathrm{Z}$. N. et al. Stabilization of $\mathrm{O}-\mathrm{O}$ bonds by $\mathrm{d}(0)$ cations in $\mathrm{Li}_{4+x} \mathrm{Ni}_{1-x} \mathrm{WO}_{6}(0 \leq \mathrm{x} \leq 0.25)$ rock salt oxides as the origin of large voltage hysteresis. J. Am. Chem. Soc. 141, 7333-7346 (2019).

17. Jacquet, Q. et al. Charge transfer band gap as an indicator of hysteresis in Li-disordered rock salt cathodes for Li-ion batteries. J. Am. Chem. Soc. 29, 11452-11464 (2019)

18. Assat, G., Glazier, S. L., Delacourt, C. \& Tarascon, J.-M. Probing the thermal effects of voltage hysteresis in anionic redox-based lithium-rich cathodes using isothermal calorimetry. Nat. Energy 4, 647-656 (2019).

19. House, R. A. et al. Superstructure control of first-cycle voltage hysteresis in oxygen-redox cathodes. Nature 577, 502-508 (2020).

20. House, R. A. et al. First-cycle voltage hysteresis in Li-rich $3 d$ cathodes associated with molecular $\mathrm{O}_{2}$ trapped in the bulk. Nat. Energy 5, 777-785 (2020)

21. Assat, G., Delacourt, C., Corte, D. A. D. \& Tarascon, J.-M. Editors' choice-practical assessment of anionic redox in Li-rich layered oxide cathodes: a mixed blessing for high energy Li-ion batteries. J. Electrochem. Soc. 163, A2965-A2976 (2016).

22. Dreyer, W. et al. The thermodynamic origin of hysteresis in insertion batteries. Nat. Mater. 9, 448-453 (2010).

23. Menil, F. Systematic trends of the ${ }^{57} \mathrm{Fe}$ Mössbauer isomer shifts in $\left(\mathrm{FeO}_{\mathrm{n}}\right)$ and $\left(\mathrm{FeF}_{\mathrm{n}}\right)$ polyhedra. Evidence of a new correlation between the isomer shift and the inductive effect of the competing bond $T-X(\rightarrow \mathrm{Fe})$ (where $X$ is $\mathrm{O}$ or $\mathrm{F}$ and $T$ any element with a formal positive charge). J. Phys. Chem. Solids 46, 763-789 (1985).

24. Saubanère, M., McCalla, E., Tarascon, J. M. \& Doublet, M. L. The intriguing question of anionic redox in high-energy density cathodes for Li-ion batteries. Energy Environ. Sci. 9, 984-991 (2016).

25. McCalla, E. et al. Understanding the roles of anionic redox and oxygen release during electrochemical cycling of lithium-rich layered $\mathrm{Li}_{4} \mathrm{FeSbO}_{6}$. J. Am. Chem. Soc. 137, 4804-4814 (2015).

26. Marcus, R. A. \& Sutin, N. Electron transfers in chemistry and biology. Biochim. Biophys. Acta Rev. Bioenerg. 811, 265-322 (1985).

27. Marcus, R. A. Electron transfer reactions in chemistry. Theory and experiment. Rev. Mod. Phys. 65, 599-610 (1993).

28. Mikkelsen, K. V. \& Ratner, M. A. Electron tunneling in solid-state electron-transfer reactions. Chem. Rev. 87, 113-153 (1987).

29. Heubner, C., Schneider, M. \& Michaelis, A. Investigation of charge transfer kinetics of Li-intercalation in $\mathrm{LiFePO}_{4}$. J. Power Sources 288, 115-120 (2015).

30. Maitra, U. et al. Oxygen redox chemistry without excess alkali-metal ions in $\mathrm{Na}_{2 / 3}\left[\mathrm{Mg}_{0.28} \mathrm{Mn}_{0.72}\right] \mathrm{O}_{2}$. Nat. Chem. 10, 288-295 (2018).

31. Mortemard de Boisse, B. et al. Intermediate honeycomb ordering to trigger oxygen redox chemistry in layered battery electrode. Nat. Commun. 7, 11397 (2016).

32. Shi, D.-R. et al. Reversible dual anionic-redox chemistry in NaCrSSe with fast charging capability. J. Power Sources 502, 230022 (2021).

33. Lee, J. et al. Unlocking the potential of cation-disordered oxides for rechargeable lithium batteries. Science 343, 519-522 (2014).

34. Yabuuchi, N. et al. High-capacity electrode materials for rechargeable lithium batteries: $\mathrm{Li}_{3} \mathrm{NbO}_{4}$-based system with cation-disordered rocksalt structure. Proc. Natl Acad. Sci. USA 112, 7650-7655 (2015).

35. Yabuuchi, N. et al. Origin of stabilization and destabilization in solid-state redox reaction of oxide ions for lithium-ion batteries. Nat. Commun. 7 13814 (2016).

36. Lee, J. et al. A new class of high capacity cation-disordered oxides for rechargeable lithium batteries: Li-Ni-Ti-Mo oxides. Energy Environ. Sci. 8, 3255-3265 (2015).

37. Chen, R. et al. Disordered lithium-rich oxyfluoride as a stable host for enhanced $\mathrm{Li}^{+}$intercalation storage. Adv. Energy Mater. 5, 1401814 (2015).

38. Radin, M. D., Vinckeviciute, J., Seshadri, R. \& Van der Ven, A. Manganese oxidation as the origin of the anomalous capacity of Mn-containing Li-excess cathode materials. Nat. Energy 4, 639-646 (2019). 
39. Li, B. et al. Thermodynamic activation of charge transfer in anionic redox process for Li-ion batteries. Adv. Funct. Mater. 28, 1704864 (2018).

40. Lee, J. et al. Mitigating oxygen loss to improve the cycling performance of high capacity cation-disordered cathode materials. Nat. Commun. 8, 981 (2017)

41. Lee, J. et al. Reversible $\mathrm{Mn}^{2+} / \mathrm{Mn}^{4+}$ double redox in lithium-excess cathode materials. Nature 556, 185-190 (2018).

42. McCalla, E. et al. Reversible Li-intercalation through oxygen reactivity in Li-rich Li-Fe-Te oxide materials. J. Electrochem. Soc. 162, A1341-A1351 (2015)

43. Song, B. et al. Understanding the low-voltage hysteresis of anionic redox in $\mathrm{Na}_{2} \mathrm{Mn}_{3} \mathrm{O}_{7}$. Chem. Mater. 31, 3756-3765 (2019).
44. Kitchaev, D. A., Vinckeviciute, J. \& Van der Ven, A. Delocalized metal-oxygen $\pi$-redox is the origin of anomalous nonhysteretic capacity in Li-ion and Na-ion cathode materials. J. Am. Chem. Soc. 143, 1908 (2021).

45. Zhang, Y. et al. Revisiting the $\mathrm{Na}_{2 / 3} \mathrm{Ni}_{1 / 3} \mathrm{Mn}_{2 / 3} \mathrm{O}_{2}$ cathode: oxygen redox chemistry and oxygen release suppression. ACS Cent. Sci. 6, 232-240 (2020).

Publisher's note Springer Nature remains neutral with regard to jurisdictional claims in published maps and institutional affiliations.

(c) The Author(s), under exclusive licence to Springer Nature Limited 2021 


\section{Methods}

Synthesis of LTFO. To prepare $\mathrm{Li}_{1.17} \mathrm{Ti}_{0.33} \mathrm{Fe}_{0.5} \mathrm{O}_{2}$, a co-precipitation method was used to obtain the $\mathrm{Ti}-\mathrm{Fe}$ hydroxide precursor. First, stoichiometric amounts of $\mathrm{TiOSO}_{4}$ (Chem Cruz) and $\mathrm{FeSO}_{4} \cdot 7 \mathrm{H}_{2} \mathrm{O}$ (Alfa Aesar; >99\%) were dissolved together in deionized water to obtain a salt solution with a $\mathrm{Ti} / \mathrm{Fe}$ concentration of $2 \mathrm{M}$. Then, the co-precipitation reaction was performed at room temperature by dropwise addition of $2 \mathrm{M} \mathrm{KOH}$ (Alfa Aesar; 99.98\%) aqueous solution and $\mathrm{Ti}$ / Fe salt solution simultaneously into $800 \mathrm{ml}$ water while maintaining the $\mathrm{pH}$ at 11 . The whole process was performed in a continuous stirred tank reactor (CSTR, Eppendorf BioFlo 320) with a stirring speed of 1,000 rpm and a solution adding speed of $1 \mathrm{ml} \mathrm{min}^{-1}$. Next, the precipitate was aged overnight and then washed with deionized water several times before transferring it to a vacuum oven and drying overnight at $80^{\circ} \mathrm{C}$. The Ti-Fe precursor $(0.5 \mathrm{~g})$ was then mixed with $2 \mathrm{~g} \mathrm{LiOH} \cdot \mathrm{H}_{2} \mathrm{O}$ (Sigma-Aldrich; $\geq 99.0 \%$ ) and dispersed into $16 \mathrm{ml}$ deionized water, after which the suspension was placed into a $20 \mathrm{ml}$ autoclave for the hydrothermal reaction at $220^{\circ} \mathrm{C}$ for $12 \mathrm{~h}$. Finally, the target LTFO product was obtained by filtering the suspension, washing the solid with water three times and then fully drying it in a vacuum oven at $80^{\circ} \mathrm{C}$.

Electrochemical characterizations. To assess the electrochemistry of LTFO, a 2032-type coin cell was applied, otherwise a Swagelok cell was used for readily recovering the powder for special ex situ characterizations. The cathode electrode was prepared by mixing $70 \mathrm{wt} \%$ LTFO powder with $20 \mathrm{wt} \%$ carbon Super P as the conductive agent and $10 \mathrm{wt} \%$ polytetrafluoroethylene (PTFE) as the binder together and making it into freestanding films. The coin cells were then fabricated in an Ar-filled glovebox, with lithium foil as the anode and a Whatman GF/D borosilicate glass fibre sheet as the separator. LP30 electrolyte was used, with a composition of $1 \mathrm{M} \mathrm{LiPF}_{6}$ in ethylene carbonate (EC)/dimethyl carbonate (DMC) in a volume ratio of 1:1. Galvanostatic cycling was applied in the electrochemistry test, unless specified otherwise. GITT measurements were performed by periodically pulsing and relaxing the cells and the details are given in the relevant places of the main text. The EIS test during relaxation was performed with a three-electrode Swagelok cell, of which the reference electrode was a copper wire dipped with a small amount of lithium. The impedance spectra were measured by applying a periodic $10 \mathrm{mV}$ potential wave with frequencies ranging from $200 \mathrm{kHz}$ to $1.4 \mathrm{MHz}$.

Structural characterizations (XRD, NPD, TEM and ICP-AES). Synchrotron XRD patterns (wavelength $\lambda=0.4579 \AA$ ) were collected via the mail-in service of the 11-BM beamline at the Advanced Photon Source at Argonne National Laboratory. NPD experiments were perfomed using the high-resolution powder diffractometer SPODI (wavelength $\lambda=1.5482 \AA$ ) at Forschungs-Neutronenquelle Heinz Maier-Leibnitz (FRM II) at the Technical University of Munich. In situ XRD was carried out using a laboratory X-ray diffractometer (BRUKER D8 Advance) equipped with a $\mathrm{Cu} \mathrm{K} \alpha$ radiation source $\left(\lambda_{\mathrm{K} \alpha 1}=1.54056 \AA, \lambda_{\mathrm{K} \alpha 2}=1.54439 \AA\right)$ and a Lynxeye XE detector. A homemade airtight cell with a Be window was used. All the Rietveld refinements of the XRD and NPD patterns were performed using the FullProf program ${ }^{46}$. ICP-AES analysis was conducted on a PerkinElmer NexION 2000 ICP mass spectrometer. The samples for ICP-AES were first dissolved in aqua regia and then diluted with deionized water in a volumetric flask to the appropriate concentration before the measurements.

TEM. TEM samples were prepared by crushing the crystals with an agate mortar and pestle in DMC and depositing drops of suspension onto a carbon film supported by a copper grid. Samples for TEM were stored and prepared in an Ar-filled glovebox. A special Gatan vacuum transfer holder was used for analysis and transportation of the samples from the Ar-filled glovebox to the TEM column to prevent interaction between the samples and air. ED patterns, HAADF-STEM images and STEM-EDX compositional maps were acquired on a probe aberration-corrected FEI Titan Themis Z 80-300 electron microscope at $200 \mathrm{kV}$ equipped with a Super-X system for EDX analysis.

OEMS measurements. Freestanding electrodes made from 70\% LTFO, 20\% carbon Super P and 10\% PTFE binder were used to perform the OEMS experiments with an in-house developed cell ${ }^{47}$. To ensure reproducibility, three OEMS experiments were run simultaneously. Two of them used a LP30 electrolyte while the other one used LP47 electrolyte (1 M LiPF 6 in EC/DEC wt. 3:7). A lithium foil anode and one piece of a Whatman GF/D glassfibre separator were used to construct the half cell. The gas signal was collected by resting the cell for $4 \mathrm{~h}$ before and after two cycles to stabilize the background signal.

In situ Mössbauer spectroscopy. Operando and in situ ${ }^{57} \mathrm{Fe}$ Mössbauer analyses were carried out in the transmission geometry in the constant acceleration mode and with a ${ }^{57} \mathrm{Co}(\mathrm{Rh})$ source with radioactivity of $500 \mathrm{MBq}$. The velocity scale $\left( \pm 4 \mathrm{~mm} \mathrm{~s}^{-1}\right)$ was calibrated at room temperature with $\alpha$-Fe foil. To avoid using thick electrodes and a longer data collection time, the absorbers were prepared using $100 \%{ }^{57} \mathrm{Fe}$. During cycling, a spectrum was collected every hour. The electrodes used contained $2-5 \mathrm{mg} \mathrm{cm}^{-2}$ LTFO mixed with $15 \%$ carbon black and $10 \%$ PTFE. The hyperfine parameters IS (isomer shift) and QS (quadrupole splitting) were determined by fitting Lorentzian lines to the experimental data. The isomer shift values were calculated with respect to that of an $\alpha$-Fe standard at room temperature. The in situ cell was similar to the one used for XAS analysis (see below). Note that the spectrum of the pristine material consisted of two sub-spectra highlighting the fact that iron environments were not identical as can be expected regarding the distribution of the nearest neighbours of iron $(\mathrm{Fe}, \mathrm{Ti}, \mathrm{Li})$ This nearest-next neighbour effect was responsible for the spectra broadening. For the sake of clarity, only the sum of the two doublets is presented ${ }^{48}$.

XAS. Ex situ Fe and Ti K-edge XAS and in situ Fe and Ti K-edge XAS spectra during relaxation were collected in transmission mode at the ROCK beamline ${ }^{49}$ of the SOLEIL synchrotron facility in France. A Si(111) channel-cut quick-XAS monochromator with an energy resolution of $0.7 \mathrm{eV}$ at $7 \mathrm{keV}$ was used. The intensity of the monochromatic X-ray beam was measured using three consecutive ionization detectors. To prepare the ex situ samples, freestanding LTFO electrode films were cycled to the desired SoC, after which they were extracted from the cell and sealed with Kapton tape and placed in air-tight X-ray transparent plastic bags under argon for protection. For the in situ relaxation measurement, an electrochemical cell equipped with Be windows ${ }^{50}$ was placed between the first and second ionization chambers. The energy calibration was performed using Fe and Ti foils placed between the second and third ionization chambers. All XAS data were processed with the Athena program ${ }^{51}$. Fourier transformation was implemented for $k^{2}$-weighted EXAFS oscillations in the $k$ range of 2.7 to $9.8 \AA^{-1}$ for both Fe and Ti K-edge spectra using a sine window function. The EXAFS fittings of Fe K-edge spectra before and after relaxation were performed in the $R$ range of 1 to $3 \AA$.

HAXPES. Ex situ HAXPES measurements were carried out at the GALAXIES beamline ${ }^{52}$ of the SOLEIL synchrotron in France. The powder-form samples were cycled to the desired SoC with Swagelok cells and then recovered, washed with DMC three times and then dried under reduced pressure overnight. The transferring of samples from the glovebox to the HAXPES chamber was always done under argon or reduced pressure to avoid air contact. A third-order reflection of a $\mathrm{Si}(111)$ double-crystal monochromator was used to obtain the photon excitation energy $h \nu=10 \mathrm{keV}$. The photoelectrons were collected and analysed using a SCIENTA EW4000 spectrometer, with an energy resolution of $0.22 \mathrm{eV}$ at $10.0 \mathrm{keV}$ photon energy for the Au Fermi edge. No charge neutralizer was required and the analysis chamber pressure was kept around $10^{-8} \mathrm{mbar}$ during the experiment. The measurements were performed using single-bunch mode to minimize the $\mathrm{X}$-ray damage to the samples.

DFT $+\boldsymbol{U}$ calculations. All the calculations performed were done with the Vienna Ab initio Simulation Package (VASP), within the generalized gradient approximation and using the Perdew-Burke-Ernzerhof potential for electron exchange and correlation energy ${ }^{53-55}$. For searching the ground-state structure of the LTFO supercell $\left(\mathrm{Li}_{14} \mathrm{Ti}_{4} \mathrm{Fe}_{6} \mathrm{O}_{24}\right)$, a genetic algorithm (GA) method was implemented. The details and the rationality for using the ground-state structure for further calculations (analysed by the cation distribution) are given in Supplementary Fig. 15. A $600 \mathrm{eV}$ energy cutoff and a $3 \times 2 \times 6 k$-point mesh were further used to optimize the ground-state structure within a spin-polarized $\mathrm{DFT}+U$ framework, where $U_{\text {eff }}=U-J$ ( $\mathrm{U}$ and $\mathrm{J}$ are the parameters for describing on-site coulomb and exchange interactions) was set to $5.3 \mathrm{eV}$ for Fe unless specified. The convergence conditions for all the calculations were set as $10^{-5} \mathrm{eV}$ for electronic loops and $0.02 \mathrm{eV}^{-1}$ for ionic loops. For Fukui function calculations, we removed 0.2 electrons from the fully relaxed pristine structure ( 222 electrons) and performed the electronic relaxation while fixing the geometric structure. We finally obtained the charge density difference using the following equation:

$$
f_{-}(r)=\rho_{N}(\boldsymbol{r})-\rho_{N-0.2}(\boldsymbol{r}),
$$

where $f_{-}(r)$ is the Fukui function, $\rho_{N}(\boldsymbol{r})$ is the charge density of the pristine structure $\left(N=222\right.$ denotes the number of electrons) and $\rho_{N-0.2}(\boldsymbol{r})$ is the charge density of the pristine structure with 0.2 electrons removed.

\section{Data availability}

All of the data supporting the findings of this study are available within the paper and the Supplementary Information. Source data are provided with this paper.

\section{References}

46. Rodríguez-Carvajal, J. Recent advances in magnetic structure determination by neutron powder diffraction. Physica B 192, 55-69 (1993).

47. Novák, P. et al. Advanced in situ characterization methods applied to carbonaceous materials. J. Power Sources 146, 15-20 (2005).

48. Li, Z., Ping, J. Y., Jin, M. Z. \& Liu, M. L. Distribution of $\mathrm{Fe}^{2+}$ and $\mathrm{Fe}^{3+}$ and next-nearest neighbour effects in natural chromites: comparison between results of QSD and Lorentzian doublet analysis. Phys. Chem. Miner. 29, 485-494 (2002).

49. Briois, V. et al. ROCK: the new Quick-EXAFS beamline at SOLEIL. J. Phys. Conf. Ser. 712, 012149 (2016). 
50. Leriche, J. B. et al. An electrochemical cell for operando study of lithium batteries using synchrotron radiation. J. Electrochem. Soc. 157, A606 (2010).

51. Ravel, B. \& Newville, M. ATHENA, ARTEMIS, HEPHAESTUS: data analysis for X-ray absorption spectroscopy using IFEFFIT. J. Synchrotron Radiat. 12, 537-541 (2005).

52. Rueff, J.-P. et al. The GALAXIES beamline at the SOLEIL synchrotron: inelastic X-ray scattering and photoelectron spectroscopy in the hard X-ray range. J. Synchrotron Radiat. 22, 175-179 (2015).

53. Perdew, J. P., Burke, K. \& Ernzerhof, M. Generalized gradient approximation made simple. Phys. Rev. Lett. 77, 3865-3868 (1996)

54. Kresse, G. \& Furthmüller, J. Efficient iterative schemes for ab initio total-energy calculations using a plane-wave basis set. Phys. Rev. B 54, 11169-11186 (1996).

55. Kresse, G. \& Joubert, D. From ultrasoft pseudopotentials to the projector augmented-wave method. Phys. Rev. B 59, 1758-1775 (1999).

\section{Acknowledgements}

XAS experiments were performed on the ROCK beamline (financed by the French National Research Agency (ANR) as a part of the 'Investissements d'Avenir' programme, reference: ANR-10-EQPX-45) at the SOLEIL Synchrotron, France under proposals \#20171234 and \#20190596. HAXPES measurements were performed on the GALAXIES beamline at the SOLEIL Synchrotron, France under proposals \#20171035 and \#20190646. This research used resources of the Advanced Photon Source, a US Department of Energy (DOE) Office of Science User Facility, operated for the DOE Office of Science by Argonne National Laboratory under Contract No. DE-AC02$06 \mathrm{CH} 11357$. Extraordinary facility operations were supported in part by the DOE Office of Science through the National Virtual Biotechnology Laboratory, a consortium of DOE national laboratories focused on the response to COVID-19, with funding provided by the Coronavirus CARES Act. Access to TEM facilities was granted by the Advance Imaging Core Facility of Skoltech. We highly appreciate help from I. Aguilar for measuring ICP-AES and help from D. Ceolin and J. Sottmann with HAXPES experiments. The authors thank D. Xia for his earlier advice and G. Assat and J. Vergnet for fruitful discussions. A.M.A and A.V.M are grateful to the Russian Science Foundation for financial support (grant 20-43-01012). J.-M.T and B.L. acknowledge funding from the European Research Council (ERC) (FP/2014)/ERC Grant-Project 670116-ARPEMA.

\section{Author contributions}

B.L. and J.-M.T. conceived the idea and designed the experiments. B.L. prepared the samples and did the electrochemical tests. M.T.S. collected and analysed the Mössbauer data. G.R. analysed the crystal structures and diffraction patterns. A.V.M. and A.M.A. performed the TEM studies and interpreted the data. A.I. and R.D. collected and analysed the XAS and HAXPES data. A.S. collected the NPD data. L.Z. performed the OEMS experiment and did the analysis. M.-L.D. developed the theoretical framework and B.L. carried out the DFT calculations. J.-M.T., M.-L.D., A.M.A. and B.L. discussed the results and wrote the paper with contributions from all authors.

\section{Competing interests}

The authors declare no competing interests.

\section{Additional information}

Supplementary information The online version contains supplementary material available at https://doi.org/10.1038/s41557-021-00775-2.

Correspondence and requests for materials should be addressed to Jean-Marie Tarascon.

Peer review information Nature Chemistry thanks Naoaki Yabuuchi and the other, anonymous, reviewer(s) for their contribution to the peer review of this work.

Reprints and permissions information is available at www.nature.com/reprints. 